Draft VERSION MAY 29, 2020

Typeset using LATEX default style in AASTeX63

\title{
AstroSat observations of GRO J2058+42 during the 2019 outburst
}

\author{
Kallol Mukerjee, ${ }^{1}$ H. M. Antia, ${ }^{1}$ And Tilak Katoch ${ }^{1}$ \\ ${ }^{1}$ Tata Institute of Fundamental Research, Homi Bhabha Road, Mumbai 400005, India
}

\begin{abstract}
We present results from AstroSat observation of the recent outburst of GRO J2058+42, an X-ray pulsar in a Be-binary system. The source was observed on April 10, 2019 by LAXPC and SXT instruments on AstroSat during its declining phase of the latest giant outburst. Light curves showed a strong pulsation of the pulsar with a period of $194.2201 \pm 0.0016 \mathrm{~s}$, and a spin-up rate of $(1.65 \pm 0.06) \times 10^{-11}$ $\mathrm{Hz} \mathrm{s}^{-1}$. Intermittent flaring was detected in light curves between 3-80 keV energy band with increase in intensity by up to 1.8 times its average intensity. Pulse profiles obtained between $3-80 \mathrm{keV}$ energy band of the pulsar showed strong dependence on energy. A broad peak was observed in the power density spectrum of the source consistently during AstroSat observations with its peak oscillation frequency of $0.090 \mathrm{~Hz}$ along with its higher harmonics, which may be due to quasi-periodic oscillations, a commonly observed phenomenon in transient X-ray pulsars, during their outburst. AstroSat observation also detected cyclotron absorption features in its spectrum corresponding to (9.7-14.4) keV, (19.3-23.8) keV and (37.8-43.1) keV. The pulse phase resolved spectroscopy of the source showed phase dependent variation in its energy and relative strength of these features. The spectrum was well fitted with an absorbed black-body, a Fermi Dirac cut-off model and alternatively with an absorbed CompTT model. Both these models were combined with a Fe-line and three Gaussian absorption lines to account for observed cyclotron resonance scattering features in the spectrum.
\end{abstract}

\section{INTRODUCTION}

Many Be-binary systems were observed earlier during outbursts which offered interesting results (Bildsten et al. 1997; Reig et al. 2011). Bright X-ray outbursts are observed in Be-binaries most likely during periastron passage of its neutron star through a circumstellar disk of its companion. Depending on amount of matter released from its companion and the geometry of the binary system, a rare as well as regular outbursts are observed during its each binary orbit (Okazaki et al. 2001, 2002; Okazaki 2016). The pulse characteristics of some of these were studied in detail during their outburst activities such as EXO 2030+375 (Parmar et al. 1989), Cepheus X-4 (Mukerjee et al. 2000), XTE J1946+274 (Paul et al. 2001). Studies on pulse characteristics offer information on the pulsar geometry and underlying mechanism for its emitted pulse profile. The shape of the emitted pulse depends on modes of accretion inflows, source luminosity, geometry of accretion column and configuration of its magnetic field with respect to an observer's line of sight (Parmar et al. 1989). Therefore, such studies offer understanding of its pulsar system and disk-magnetospheric interaction during the process of mass accretion, which affects its emitted radiation. Quasiperiodic oscillations (QPO) were detected from many Be-binaries earlier, such as A0535+262 (Finger et al. 1996, 1998), EXO2030+375 (Angelini et al. 1989), 4U0115+63 (Soong \& Swank 1989; Heindl et al. 1999; Dugair et al. 2013), V0032+53 (Qui et al. 2005). Studies of QPOs offer rich information about accretion torque onto the neutron star, thermodynamic properties of the inner accretion disk and electrodynamics of disk-magnetospheric interaction of the neutron star. Details on sources with observed QPOs, their frequencies and other features along with pulsar spin frequencies etc. are given in tabular form by Devasia et al. (2011); Ghosh (1998); Mukerjee et al. (2001). Some of these transient Be-binary pulsars such as A0535+26 $(50 \mathrm{mHz}, 9.7 \mathrm{mHz}), 1 \mathrm{~A} 1118-61(92 \mathrm{mHz}, 2.5 \mathrm{mHz})$, XTE J1858+034 (110 mHz, $4.53 \mathrm{mHz})$, EXO 2030+375 (200 mHz, $24 \mathrm{mHz})$, SWIFT J1626.6-5156 (1000 mHz, $65 \mathrm{mHz})$, XTE J0111.2-7317 (1270 mHz, $32 \mathrm{mHz})$ as well as a persistent Be-binary, X-per $(54 \mathrm{mHz}, 1.2 \mathrm{mHz})$ and an OBtype binary, 4U 1907+09 $(69 \mathrm{mHz}, 2.27 \mathrm{mHz})$ showed higher QPO frequency than their respective spin-frequency as

kmukerji@tifr.res.in 
mentioned in order inside parenthesis (Devasia et al. 2011). These cover an interestingly wide range of QPO frequencies between $50-1270 \mathrm{mHz}$ for these pulsars. Studies of cyclotron absorption features, if present in the spectrum, enable us to determine the strength of the surface magnetic field of the neutron star and offers an insight into the line producing region, structure of accretion column and its geometry (Staubert et al. 2019). The cyclotron absorption features thus provides an important diagnostic probe for detailed studies of the neutron star binaries since its discovery in the spectrum of Her X-1 (Truemper et al. 1978). There are several reports on the detection of cyclotron absorption features in the spectrum of many Be-binaries starting at a lower energy from $\sim 10 \mathrm{keV}$ (DeCesar et al. 2013; Jun et al. 2012 ) to a higher energy at $\sim 100 \mathrm{keV}$ (La Barbera et al. 2001). Detailed compilation of such sources and studies are reported by Staubert et al. (2019) and Maitra (2016). It is observed from detailed studies that some sources show a wide variation in its cyclotron-line energy with respect to its pulse-phase, source luminosity and with time, such as Vela X-1, Cen X-3 and Her X-1 (Staubert et al. 2019). These interesting properties help in understanding the nature of these sources and also offer an insight into their underlying physical properties governing such changes.

The high mass X-ray binary GRO J2058+42 is a transient X-ray pulsar which was first discovered by BATSE on board CGRO during its giant outburst in September-October, 1995. The outburst lasted for about 46 days which peaked at $300 \mathrm{mCrab}$ intensity (Wilson et al. 1998). The spin period of its neutron star decreased from $198 \mathrm{~s}$ to $196 \mathrm{~s}$ during its 46 days outburst (Wilson et al. 1998). This outburst was subsequently followed by 5 more bursts of lower intensity of about $15 \mathrm{mCrab}$ and of lesser duration of 15 days observed at an interval of about 110 days. Additional shorter outbursts with peak intensity of about $8 \mathrm{mCrab}$ were detected by BATSE, halfway between the first four outbursts of 15-20 mCrab (20-50 keV) intensity. During early 1998, two outbursts of lower intensity were observed with PCA and HEXTE on-board RXTE (Wilson et al. 2005). There were, however, no reports of any giant outburst from the source. Thus, GRO J2058+42 showed a very rare giant outburst activity, only twice so far, since its discovery. The first was detected by BATSE (Wilson et al. 1998, 2005) and the most recent one was detected by Swift-BAT (Barthelmy et al. 2019; Kennea et al. 2019) and Fermi-GBM (Malacaria et al. 2019) in March 2019. GRO J2058+42 was suggested earlier to be a high mass X-ray binary due to its observed properties. It was subsequently confirmed having a Be-star companion through optical observations (Reig et al. 2005). A very limited details are known so far about this source, due to its rare intense outbursts.

AstroSat observed the source on April 10, 2019 during the declining phase of the second giant outburst, for studies of some of the above described properties of a typical Be-binary pulsar. In this work, we present results from our studies on spectral and timing properties of GRO J2058+42 using data from AstroSat observation. Incidentally, there were no reports on detection of any cyclotron absorption features from the earlier outbursts of GRO J2058+42. We, therefore in particular, wish to check for possible detection of cyclotron absorption features in the spectrum and presence of QPOs. After this manuscript was submitted, Molkov et al. (2019) have reported detection of cyclotron absorption feature using NuSTAR observation of the source during the same outburst. The rest of the paper is organized as follows: Section 2 describes the observations and data analysis including software tools used, Section 3 describes the results and its implications are discussed in Section 4. Finally, Section 5 gives the conclusions from this study.

\section{OBSERVATIONS AND DATA ANALYSIS}

GRO J2058+42 had its rare and long outburst during March-May, 2019 as its latest event, as seen in the SwiftBAT intensity curve shown in Figure 1. The light curve was downloaded from Swift-BAT light curves Archive ${ }^{1}$ and relevant details about these curves are described by Krimm et al. (2013). As there was a data gap close to the peak of the outburst, the peak of the outburst was approximately determined by fitting a cubic B-spline with 32 uniformly distributed knots to the data while data points with large errors were dropped. It enabled us to determine peak intensity and its corresponding time which was found to be at around MJD 58574.8 with its peak intensity reaching to about $256 \mathrm{mCrab}$ (Swift-BAT $0.0564 \mathrm{cts} \mathrm{cm}^{-2} \mathrm{~s}^{-1}$ ) in the $15-50 \mathrm{keV}$ energy band. When we compared this giant outburst with the earlier outburst, it turns out that, the main flare of this giant outburst lasted for about a similar duration of 46 days (Wilson et al. 1998) and was followed by a relatively weaker and a much narrower secondary flare as seen in Figure 1. There is also a small burst about 110 days before the main burst as seen in the swift-BAT light curve (Figure 1). CGRO-BATSE observed the earlier giant outburst with a peak intensity of about $300 \mathrm{mCrab}$ (Wilson et al. 1998, 2005), comparable to that of its latest outburst which was also followed by a secondary flare observed after about 52 days.

${ }^{1}$ https://swift.gsfc.nasa.gov/results/transients/index.html 


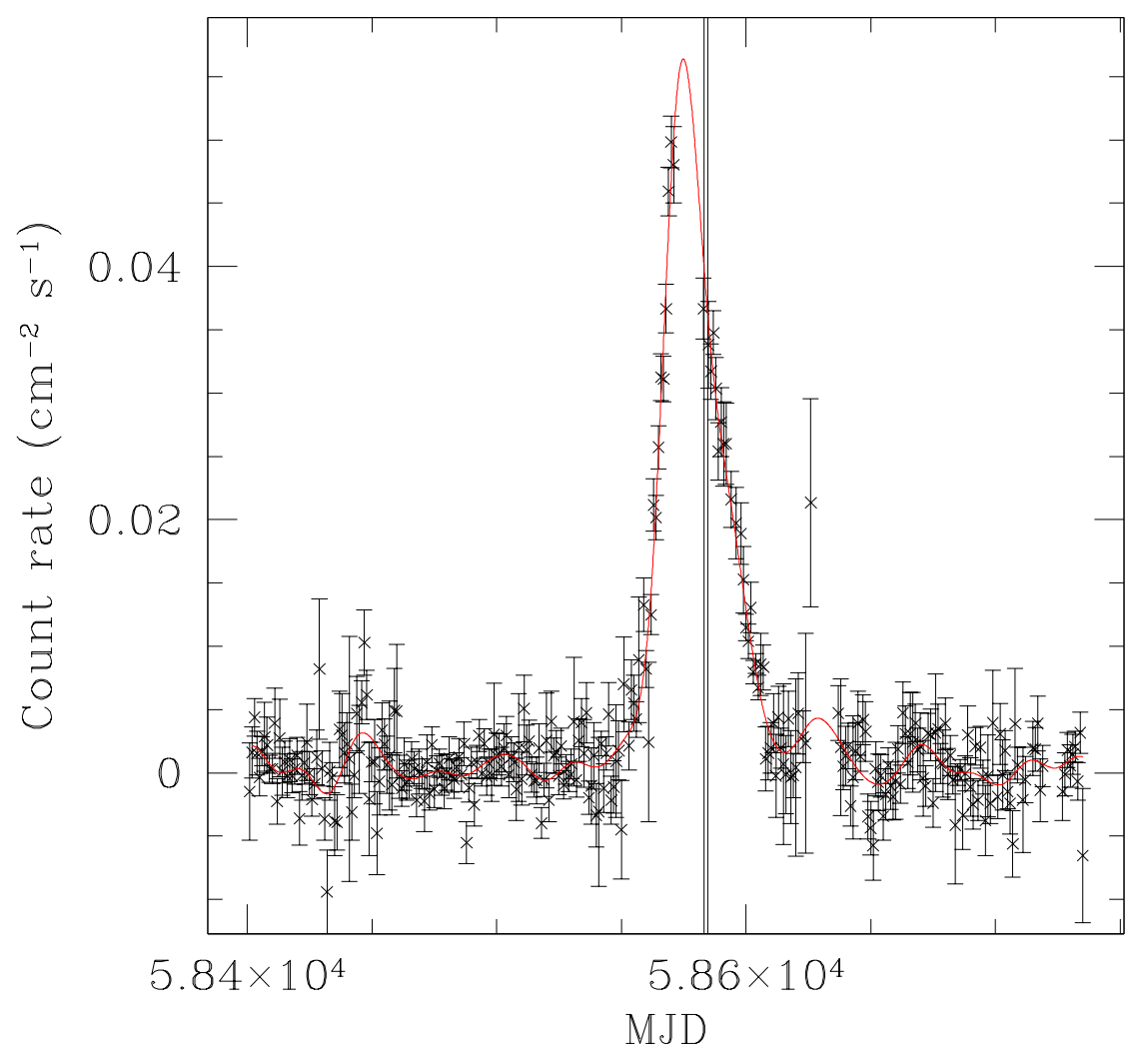

Figure 1. Intensity variation in the $15-50 \mathrm{keV}$ energy band as observed by Swift-BAT during source outburst is shown by black points while the continuous line shows a fit to the light curve with cubic B-splines using 32 uniformly distributed knots. The time covered by AstroSat Observation is marked by two vertical lines.

AstroSat observed the source on April 10, 2019 during the declining phase of the outburst as marked in the Figure 1 from MJD 58583.11 to MJD 58584.67. It was about 8-days after the peak of the outburst, when source intensity had decreased to about $170 \mathrm{mCrab}$, which is about $66 \%$ of its peak intensity as obtained above. The LAXPC was used as the primary instrument for this observation with a total effective exposure of $57 \mathrm{ks}$. Details of LAXPC instruments on board AstroSat and its operation modes are described in detail by Antia et al. (2017). During the observation only one LAXPC detector, i.e., LAXPC20 was working properly. LAXPCs were operated in its event-analysis mode. LAXPC10 was operating at a very low gain and it barely detected pulsation in the source. LAXPC20 detected an average source count-rate of $225.6 \pm 0.1 \mathrm{cts} \mathrm{s}^{-1}$ in the $6-70 \mathrm{keV}$ energy band. The SXT was operated in its photon counting mode during the whole observation, with an effective exposure of about $21 \mathrm{ks}$ and detected an average countrate of $3.78 \pm 0.01 \mathrm{cts} \mathrm{s}^{-1}$ in the $0.8-7 \mathrm{keV}$ energy band. The SXT instrument along with its operational modes are described in detail by Singh et al. (2016). Thus useful data from the AstroSat SXT and LAXPC20 were used for timing and spectroscopic analysis, covering a total energy band of $0.8-80 \mathrm{keV}$. The AstroSat observation was made under a Target of Opportunity (TOO) proposal with the observation ID of 20190410_T03_098T01_9000002836. AstroSat data from the proposal are available from the AstroSat Data Archive ${ }^{2}$.

LAXPC data were analyzed using LAXPC pipeline software version 3.0. The LAXPC software can be downloaded from the LAXPC site $^{3}$. The software takes level-1 data files as input and also utilizes calibration and background files to generate level-2 data products, like light curve and spectrum. The background files are available with the software, which also gives a suitable recommendation for selection of a background and associated response files which may be used for data product generation and analysis. The background observation during April 19, 2019 was used to estimate the contribution from the source. Events were extracted from all main anodes from all the layers of LAXPC20 for this

\footnotetext{
${ }^{2}$ https://astrobrowse.issdc.gov.in/astro_archive/archive/Home.jsp

${ }^{3}$ http::www.tifr.res.in/ ${ }^{\sim}$ astrosat_laxpc/LaxpcSoft.html
} 
analysis. The pipeline software was executed with default values. However, selection of appropriate energy channels were made for deriving light curves for a particular energy band. The pipeline software also corrects for any shift in the gain based on calibration data and generates corrected spectrum and background subtracted light curves of the source within its set energy band.

The SXT data analysis pipeline software version AS1SXTLevel2-1.4b was used for a set of default parameters in this analysis. Spectral response file and auxiliary response files of the SXT including background files are offered along with data analysis tools and can be downloaded from the SXT payload operation center site ${ }^{4}$. Updated CALDB files are directly linked to the pipeline processing software. All photon events were extracted by including a circular area covering a region of interest of 6 arc minutes radius with respect to the source center. All grades between 0-12 were considered for selection of photon events. The pipeline analysis software takes level-1 data as its input along with other relevant files for the analysis of events in steps to finally produce clean and calibrated event lists. The final data products such as spectrum, light curve and image are produced by applying various default filters and appropriate screening of the data. From calibrated clean events, one can produce spectrum and light curves in different energy bands covering 0.8-7.0 keV. The 'Xselect' tool version 2.4c was used for screening the data. The solar system barycentric corrections were applied to the time series for both SXT and LAXPC data to correct for arrival time delays of events prior to its detailed timing analysis, using a tool 'as1bary' ${ }^{5}$, developed by the AstroSat science support cell.

Heasoft version 6.14 was used for analysis of this data. The standard software XRONOS 5.22 was used for timing analysis; lcurve for generation of combined light curves, efsearch for determination of spin-period of the pulsar, powspec for computing power density spectrum etc.. Standard spectral analysis tool XSPEC version 12.8.1 was used for fitting spectral data with combination of appropriate models as described below. Results obtained from these analysis are presented in the next section.

\section{RESULTS}

\subsection{X-ray light curve and folded pulse profiles}

Extracted light curve of GRO J2058+42 showed regular and strong pulsations along with variation in its intensity. A light curve segment of LAXPC20 with $10 \mathrm{~s}$ binning is shown in the left panel of Figure 2. Source intensity variation between the 3-80 keV energy band for the complete observation duration of LAXPC20 is shown in the right panel of Figure 2, obtained by binning the data with its established spin-period. A straight-line over the binned light curve shows its average intensity. Intensity variations were clearly seen for the complete duration of AstroSat observation. Observed source count rate varied from $230 \mathrm{cts} \mathrm{s}^{-1}$ to about $520 \mathrm{cts} \mathrm{s}^{-1}$ with an average count rate of $294 \mathrm{cts} \mathrm{s}^{-1}$. Intermittent flaring and dips were also seen in the light curve which is a typical intrinsic property of a Be-binary. During the flares, intensity increased by up to 1.8 times the average intensity.

The pulsar spin period was derived after applying corrections to pulse arrival time delays with respect to solar system barycenter. The average pulsar period was initially derived with epoch folding and verified using Lomb-Scargle periodogram, on full AstroSat observation data. Spin-period of the pulsar was found to be $194.180 \pm 0.001 \mathrm{~s}$ with 1- $\sigma$ confidence limit, during AstroSat observation. Total duration of the observation time was then divided into 5 intervals to estimate spin-up rate of the pulsar during AstroSat observation. The pulsar spin-period was determined for each data interval separately along with its estimated error. These periods were plotted with respect to mid-value of respective interval in MJD. An average spin-up rate of $(1.71 \pm 0.14) \times 10^{-11} \mathrm{~Hz} \mathrm{~s}^{-1}$ was derived by fitting a straight line to these 5 data points, along with its error estimated with $90 \%$ confidence limit. Then starting with these initially measured values, the spin period at the beginning of observation at $t=t_{0}$ and its time derivative were accurately determined by correcting the phase using

$$
\phi(t)=\phi_{0}+\nu_{0}\left(t-t_{0}\right)+\dot{\nu} \frac{\left(t-t_{0}\right)^{2}}{2}
$$

where $\phi$ is the phase (in range $0-1$ over the period), $\phi_{0}$ is the phase at initial time, $t_{0}, \nu_{0}$ is the spin frequency at initial time and $\dot{\nu}$ is its time derivative. The fit was performed by fitting a periodic signal with 20 harmonics of the basic period using Eq. 2,

$$
c(t)=c_{0}+\sum_{k=1}^{N}\left(a_{k} \sin (2 \pi k \phi)+b_{k} \cos (2 \pi k \phi)\right),
$$

${ }^{4}$ http::www.tifr.res.in/ astrosat_sxt/index.html

${ }^{5}$ http://astrosat-ssc.iucaa.in/?q=data_and_analysis 

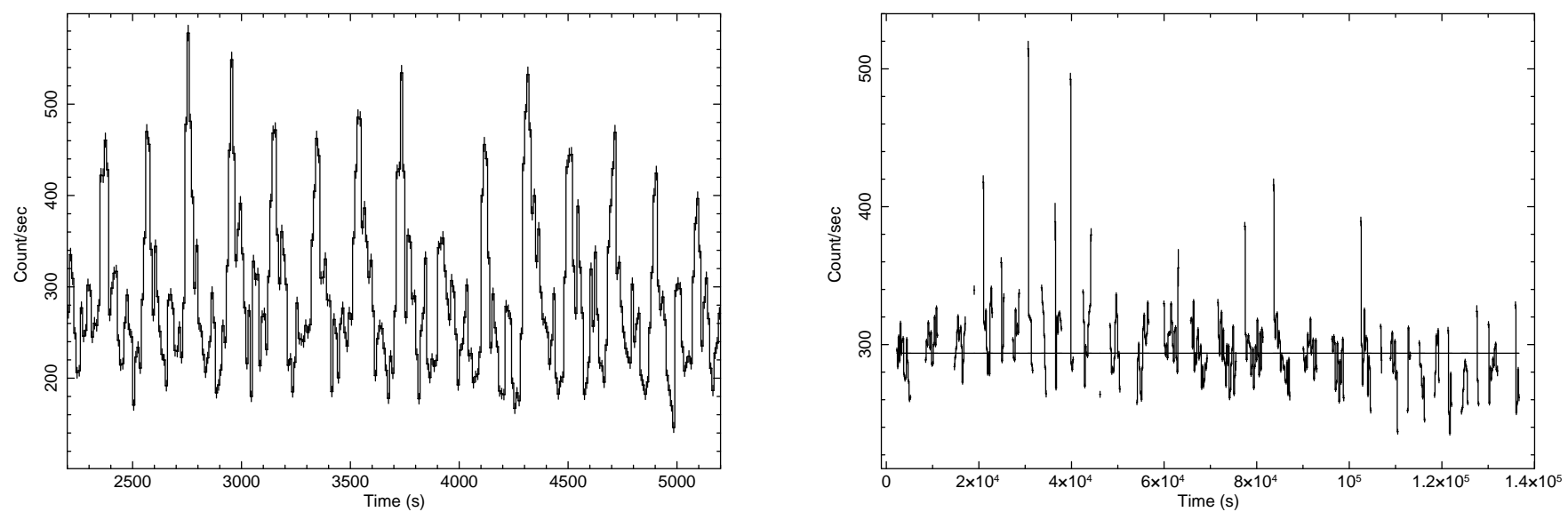

Figure 2. Light curves derived from LAXPC20 in the 3-80 keV energy band are shown, as a segment with $10 \mathrm{~s}$ binning (left panel) and also for the entire AstroSat observation with a binning of spin-period of the pulsar (right panel). The reference time $t_{0}$ is MJD 58583.10868148 for both these figures. The horizontal line on the right panel shows the average source count rate of 294 counts $\mathrm{s}^{-1}$.

where $c(t)$ is the observed count rate, $N$ is the number of harmonics included in the fit and $c_{0}, a_{k}, b_{k}$ are the coefficients fitted, apart from $\nu_{0}$ and $\dot{\nu}$. The light curve was obtained with a time-bin of $1 \mathrm{~s}$. The best fit values for $\nu_{0}$ and $\dot{\nu}$ were determined by varying both parameters to minimize $\chi^{2}$ deviation of the light curve from the model (Eq. 2). Various values of $N$ were tried and $N=20$ was found to be adequate to account for all pulsation signal. In principle, it is possible to use the F-test to decide the required value of $N$, which gave a value of $N=14$ with a probability threshold of 0.05 , but some components after $k=15$ were also significant. Beyond $N=20$, the next few components were not found to be significant. That is why this value was adopted. To find the errors in the fitted values of $\nu$ and $\dot{\nu}$, we performed a Monte Carlo simulation by perturbing $c(t)$ and repeating the process for 4000 different realization of noise to find the distribution of parameter values and using this distribution, we found the $90 \%$ confidence limits for the parameters. The same program was used to generate the pulse-profiles as well as the GTI intervals for different phase intervals using the fitted values of parameters. The GTI values were then used to calculate the spectra for different phase intervals for phase resolved spectroscopy. The same program was also used to generate the light curve after subtracting the pulse profile as defined by Eq. 2 to filter out the contribution of coherent pulsation in the resulting power density spectrum.

The fitted value of the period at $t=t_{0}$ corresponding to MJD 58583.10868148 was found to be $194.2201 \pm 0.0016$ s and $\dot{\nu}=(1.65 \pm 0.06) \times 10^{-11} \mathrm{~Hz} \mathrm{~s}^{-1}$, where the error bars denote the $90 \%$ confidence limits. The orbit of the binary system is not known and the orbital motion can also contribute to the period variation during the observation. However, the Fermi-GBM observations during this outburst ${ }^{6}$ suggests that the period variation was restricted to the duration of the giant outburst, and the change in $\dot{\nu}$ during the outburst showed a good correlation with intensity, with a correlation coefficient of about 0.93 . This implies that the variation in period is largely due to accretion during the outburst.

Light curves were extracted corresponding to different energy bands and respective pulse profiles were derived by folding corresponding light curves in 64 bins with its derived spin-period of the neutron star at the beginning of the epoch and its derivative. Two cycles of all such pulse-profiles are shown in Figure 3 for clarity. Folded pulse-profiles showed variation with respect to different energy bands. The average pulse-profile covering full 3-80 keV energy band is also shown at the bottom panel of the Figure 3, for reference and relative comparison. We noticed that pulse profiles at lower energies showed pronounced and multiple-pulses which gradually merged into a double-pulse and then in a single asymmetric broad-pulse at higher energies. The RMS pulse fraction measurements and source intensity with respect to energy is shown in Figure 4. RMS pulse fraction showed energy dependent variation, which showed increase from about $20 \%$ to $30 \%$ between $3-20 \mathrm{keV}$ while at higher energies it is nearly constant. Thus pulse profiles showed remarkable variability in shape and its pulse-fraction with respect to considered energy bands between $3-80 \mathrm{keV}$.

\footnotetext{
${ }^{6}$ https://gammaray.msfc.nasa.gov/gbm/science/pulsars/lightcurves/groj2058.html
} 


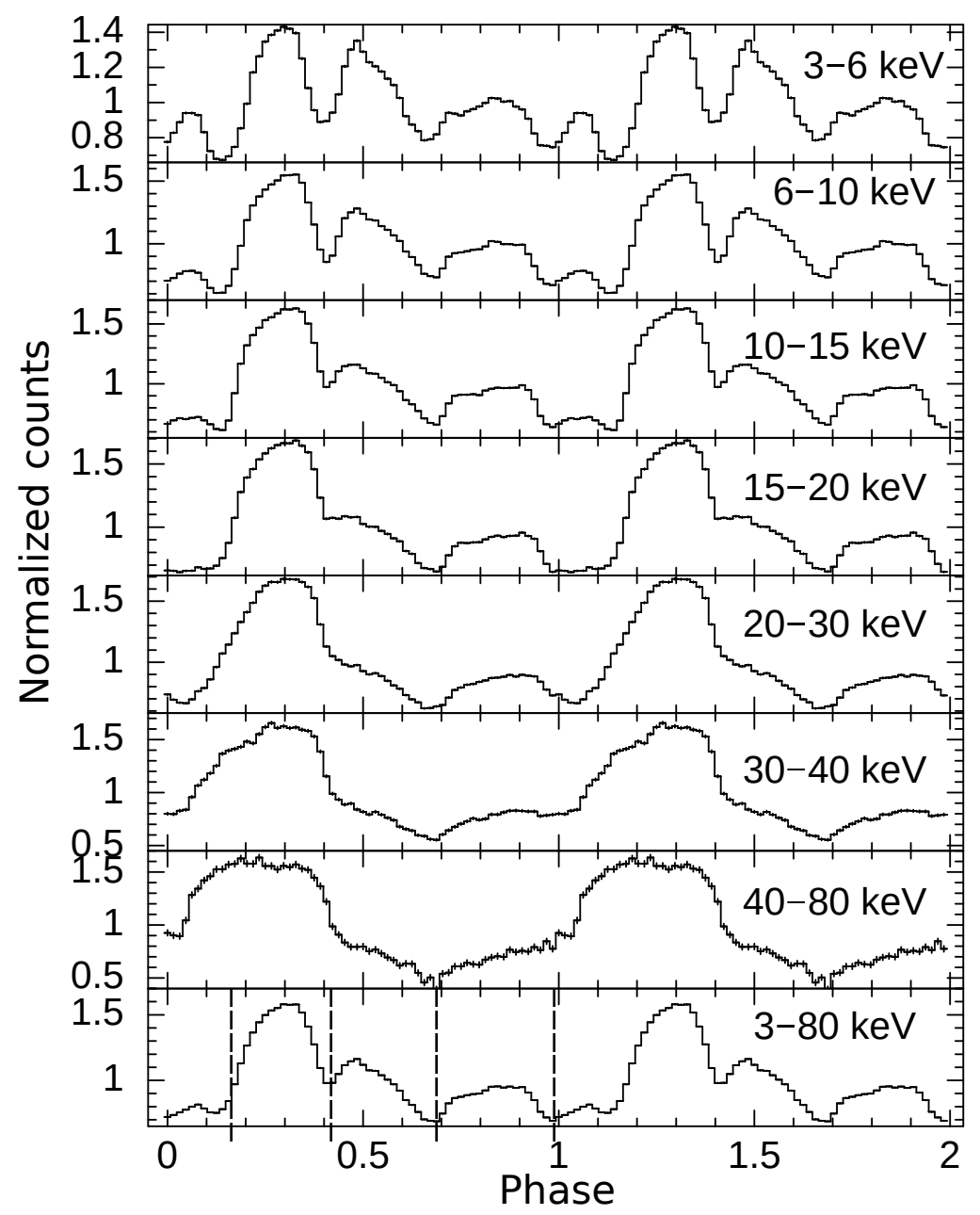

Figure 3. Folded pulse profiles derived from LAXPC20 at different energy bands covering $3-80$ keV. The vertical lines in the lowest panel mark the four divisions of pulse period used in phase resolved studies.

Pulse-profiles derived from recent AstroSat observations can be compared with earlier RXTE observation at much lower source intensity of about $10 \mathrm{mCrab}$. There is a general similarity in shape but structures are seen much more clearly than those reported from November 28, 1996 RXTE observation between 2-60 keV during its earlier lower intensity outburst (Wilson et al. 1998). For the source intensity of about $17 \mathrm{mCrab}$, the RMS pulse fractions were measured at different energy bands between 2-20 keV from the RXTE PCA observations of February 4, 1998. These were estimated as $11.7 \pm 2.6 \%(2-4 \mathrm{keV}), 19.1 \pm 4.1 \%(4-9 \mathrm{keV})$ and $27.2 \pm 5.9 \%(9-20 \mathrm{keV})$. This can be compared with the recent observation by AstroSat LAXPC20, which gives $15.6 \pm 3.3 \%(3-4 \mathrm{keV}), 22.0 \pm 4.7 \%(4-9 \mathrm{keV})$ and $28.6 \pm 6.1 \%(9-20 \mathrm{keV})$ respectively. The RXTE/PCA and AstroSat/LAXPC20 measurements show that these are comparable within respective error limits, although source intensity was an order of magnitude higher during AstroSat observation. Thus, the pulsar does not show drastic change in shape of pulse profiles and pulse-fractions with change in source luminosity in this range.

\subsection{Power density spectrum and detection of a QPO feature}

The source light curve along with its background between 3-30 keV energy band with a bin time of 1.0 second was used to derive power density spectrum. The XRONOS tool 'powspec' was used for this purpose. The normalization parameter value of -2 was selected so that power density spectra were normalized such that their integral gave the squared RMS fractional variability. Therefore, the power was expressed in the units of $(\mathrm{RMS})^{2} \mathrm{~Hz}^{-1}$ and the expected white noise level was subtracted, to obtain the RMS fractional variability of the time series. For such a normalized power density spectrum where a QPO profile was modeled by a Lorentzian, the strength of a QPO signal was described by its fractional root-mean-squared (RMS) amplitude, which is proportional to its integrated power which contributes 


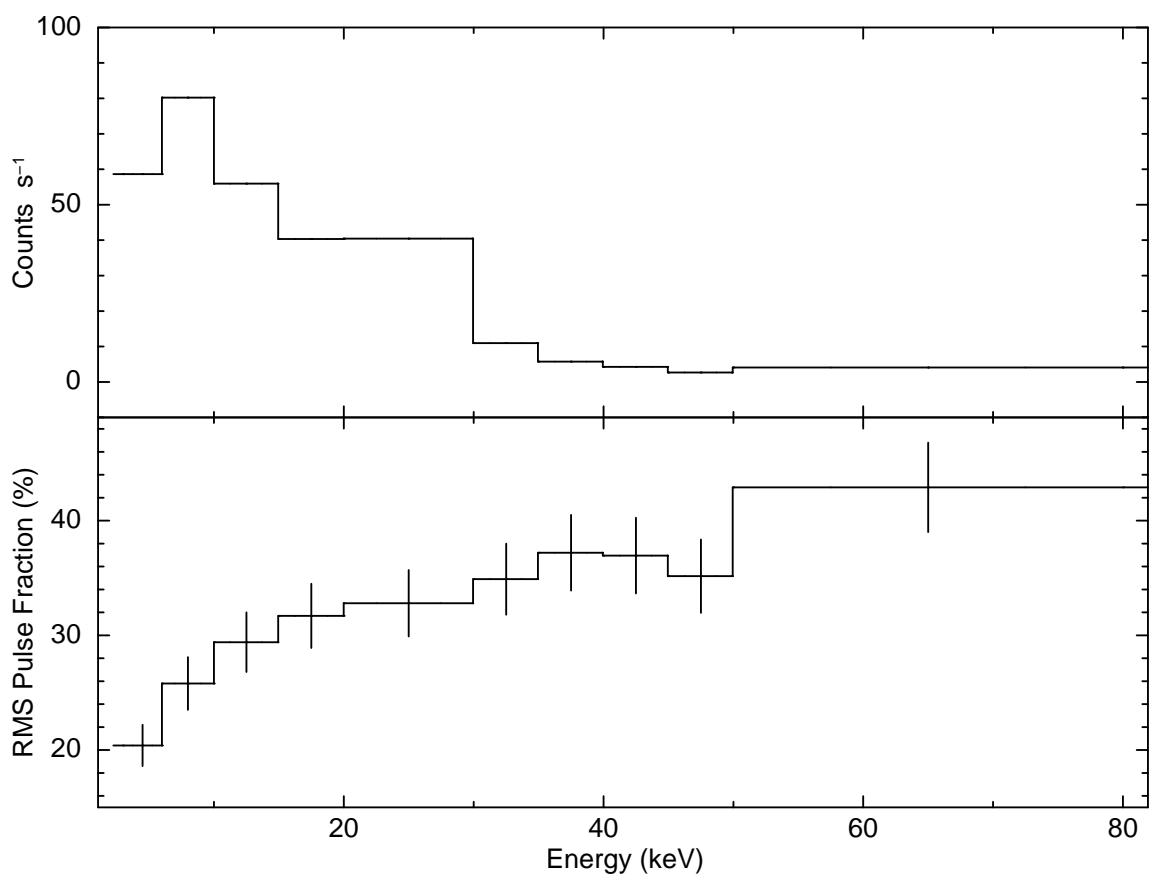

Figure 4. Count rate and RMS pulse fraction derived from LAXPC20 at different energy bands covering 3-80 keV.

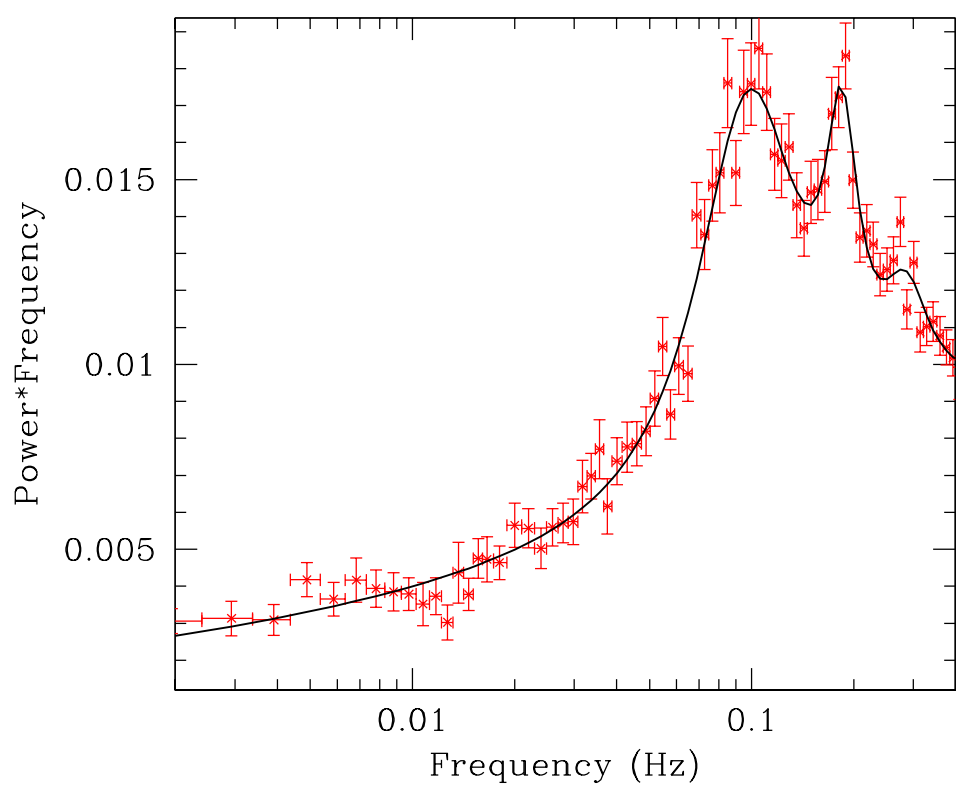

Figure 5. Power density spectrum derived from LAXPC20 data between 3-30 keV covering the observation duration is shown. A prominent QPO feature is observed at $0.09 \mathrm{~Hz}$ along with its 2 harmonics when signal from coherent spin-frequency of neutron star was removed from the time series.

to its over all power density spectrum and often expressed in percent (Wang et al. 2014). The integrated power can be computed from the area under the Lorentzian profile. The amplitude of the Lorentzian multiplied by its FWHM and $\pi / 2$ would determine the area under the profile in units of $\mathrm{RMS}^{2}$, hence its square-root would give the RMS amplitude of the QPO signal. The power density spectrum was therefore, derived considering above normalization and a total of 1024 bins per interval and total a 73 intervals in a frame were considered for the purpose of obtaining the power density spectrum. However, out of a total of 73 intervals, 16 intervals with less than $50 \%$ data, were rejected for a 
Table 1. Power density spectrum model parameters

\begin{tabular}{ccccc}
\hline \hline Parameter & Value & $\begin{array}{c}\text { Amplitude } \\
(\mathrm{RMS} \%)\end{array}$ & $\begin{array}{c}\text { F-TEST } \\
\text { FAP }\end{array}$ & $\begin{array}{c}\text { Detection } \\
\text { Significance }(\sigma)\end{array}$ \\
\hline Power Index & $-0.78 \pm 0.08$ & & & \\
Normalization (K) (at 1 Hz) & $0.010 \pm 0.001$ & & \\
& & & \\
QPO- $f_{0}$ Centroid LC (Hz) & $0.090 \pm 0.003$ & $12.1 \pm 1.0$ & $2.7 \times 10^{-11}$ & \\
Sigma LW (Hz) & $0.081 \pm 0.012$ & & & \\
Normalization LN & $0.116 \pm 0.008$ & & & \\
& & & & \\
QPO- $f_{1}$ Centroid LC (Hz) & $0.183 \pm 0.004$ & $5.2 \pm 0.9$ & $1.4 \times 10^{-7}$ & \\
Sigma LW (Hz) & $0.048 \pm 0.015$ & & & \\
Normalization LN & $0.036 \pm 0.006$ & & & \\
& & & & \\
QPO- $f_{2}$ Centroid LC (Hz) & $0.280 \pm 0.017$ & $4.2 \pm 1.5$ & $2.2 \times 10^{-4}$ & \\
Sigma LW (Hz) & $0.11 \pm 0.07$ & & & \\
Normalization LN & $0.010 \pm 0.003$ & & & \\
\hline$\chi_{\text {red }}^{2}$ (dof) & $1.08(62)$ & & & \\
\hline
\end{tabular}

Note-Errors with $90 \%$ confidence range for each parameter. FAP-False alarm probability.

default window selection. The data gaps were padded with zeros as its default option. A geometric re-binning of 1.04 was applied for generation of power density spectrum to improve on statistical fluctuation at higher frequencies.

The average power density spectrum derived as above showed spin frequency along with its several harmonics. It also showed a broad peak around $0.09 \mathrm{~Hz}$ and its 2 harmonics, which were identified as a QPO. The QPO signal was found to be better in the 3-30 keV energy band, compared to a higher energy band, hence power spectra are presented for this energy band. For confirming the presence of QPO, we removed the contribution of coherent pulsation signal by subtracting the fitted pulse profile with 20 harmonics (Eq. 2) from the time series data as described in section 3.1. Power density spectrum was then derived from this time series. The power density spectrum showed a clear detection of a QPO feature at $0.090 \pm 0.003 \mathrm{~Hz}$ along with its 2 harmonics. As the pulsation signal was removed from the time series data, power density spectrum did not show any coherent pulsations as was seen earlier. However, the continuum defined by the power law-index were found to be consistent within the $90 \%$ error limit, when coherent pulsation signal was present (power-index $=-0.89 \pm 0.09$ ) and when pulsation signal was removed, (power-index $=-0.78 \pm 0.07$ ). The observed QPO and its 2 higher harmonics defined by Lorentzian function were also found consistent within their parameter uncertainty. The power density spectrum was modeled using a power-law in combination with 3 Lorentzian functions to model QPO and its two higher harmonics. The model parameter values and QPO-frequency and its corresponding 2 harmonics as derived from the fitted model are listed in the Table- 1 . This is to mention here that since many intervals of data were averaged for generating the power density spectrum, therefore, $\chi^{2}$ test for the fitted model does not introduce significant biases as it is well known that in this case the power density spectrum has distribution close to the normal distribution (Barret \& Vaughan 2012). The model was fitted and parameter values were established from the power density spectrum when its y-axis was normalized and expressed in the units of $(\mathrm{RMS})^{2} \mathrm{~Hz}^{-1}$ and frequency along the $\mathrm{x}$-axis. However, the power density spectrum is shown in Figure 5 with y-axis multiplied by corresponding frequency to show prominence of the QPO and its 2 harmonics. The Lorentzian function $L(f)$ used in the model is defined as:

$$
L(f)=\frac{\mathrm{LN}}{1+\left(\frac{2(f-\mathrm{LC})}{\mathrm{LW}}\right)^{2}},
$$

where parameters LN, LC and LW represent its peak amplitude, centroid frequency and its full width at half maximum, respectively. 

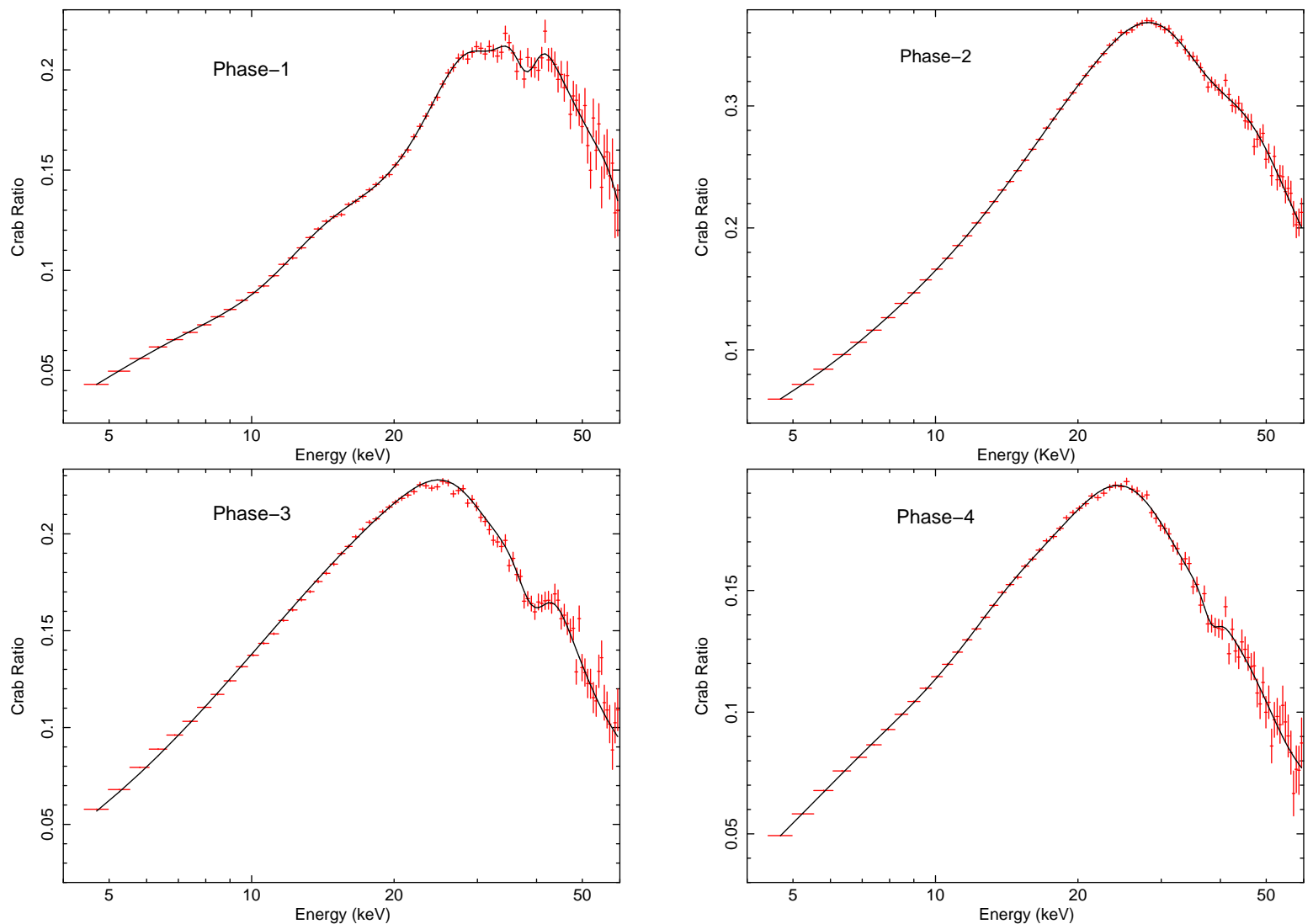

Figure 6. Ratio of spectral counts of the source at different energies with respect to Crab spectral counts is shown corresponding to 4 different pulse-phases of the pulsar (as marked in Figure 3) derived from LAXPC20 spectral data between 4-60 keV energy band. The continuous line shows the fit with a combination model of a polynomial of degree 3 in energy and Gaussians to define dips in the ratio.

An alternate model, broken-power-law along with 3 Lorentzian functions was also fitted to the same power density spectrum for comparison. The reduced $\chi^{2}$ value of 1.12 was found for the 60 degrees of freedom (dof), as compared with power-law model along with 3 Lorentzian functions which yields the reduced $\chi^{2}$ of 1.08 for 62 dof. Thus, power-law model resulted in a better fit, compared to broken power-law model. A break frequency of $0.31 \pm 0.01 \mathrm{~Hz}$ was obtained from broken-power law model when fitted to the power density spectrum. The break frequency is much higher than the observed QPO frequency of $0.09 \mathrm{~Hz}$ and hence it cannot mimic the QPO.

Thus, RMS amplitudes determined from the averaged power density spectrum, by fitting a power-law and 3 Lorentzian for QPO and its 2 harmonics are also given in Table-1. The statistical significance of detected QPOs was established using the F-test. This was done by determining the difference in $\chi^{2}$ with and without the inclusion of a Lorentzian function, used for modeling detected QPO in the power density spectrum. Similar approach was followed for its other 2 harmonics, individually. The false alarm probability (FAP) and detection significance of QPO expressed in terms of $\sigma$ for an equivalent normal distribution is computed and listed in Table- 1 for QPO and its 2 harmonics. It turns out that the QPO frequency and the two harmonics are significant. It is observed that apart from the first harmonic the other two peaks are rather broad.

\subsection{Spectrum and detection of cyclotron absorption features}

RXTE derived the first X-ray spectrum of the source during the earlier outburst of 1996 covering energy band between 2.7-50 keV. The spectrum was modeled adequately well with absorbed thermal bremsstrahlung (Phabs*bremss). However, there was, no report on any detection of cyclotron absorption feature from RXTE observations. Therefore, to check for the presence of a cyclotron absorption feature in the spectrum we took the ratio of counts in the spectrum of source to that in the Crab spectrum which was observed by LAXPC20 of AstroSat and the results for the phaseresolved spectra are shown in Figure 6. The overall pulse phase was divided into 4 intervals covering structures of the 

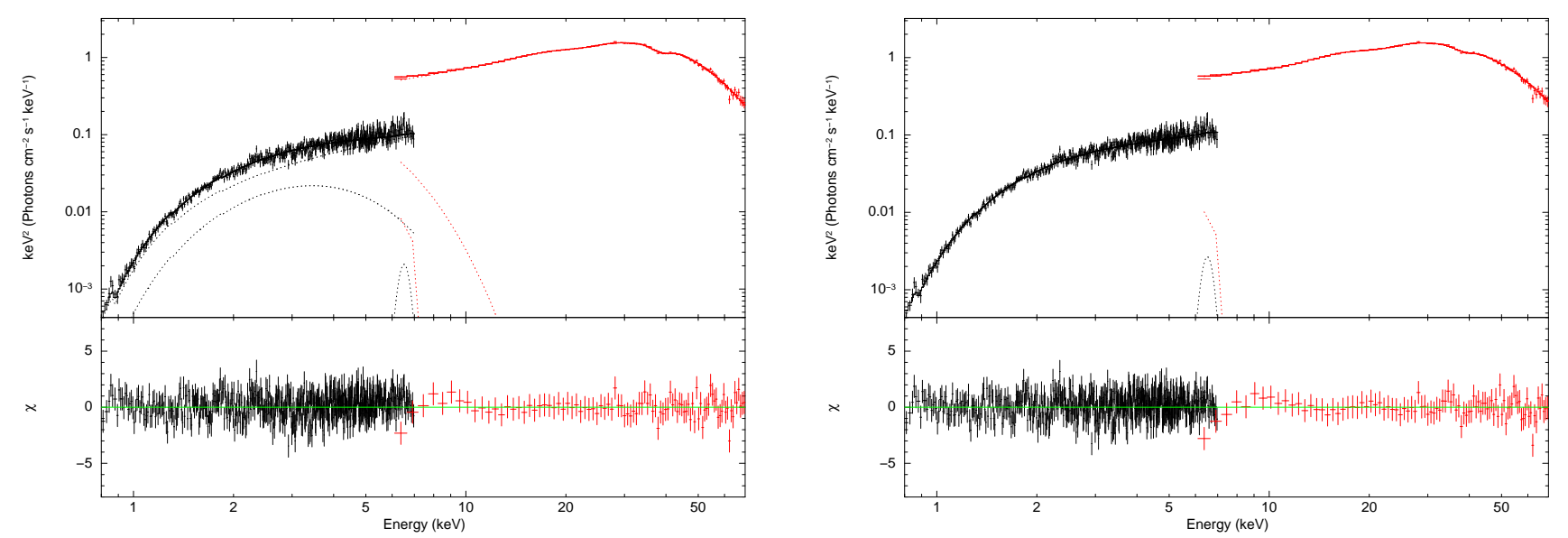

Figure 7. Spectrum fitted with combined model; an absorbed Fermi-Dirac cutoff model with a black-body a Gaussian emission line and 3 Gaussian absorption lines (Model 1, left panel), and an absorbed CompTT with a Gaussian emission line and 3 Gaussian absorption lines (Model 2, right panel) are shown.

folded pulse profile as shown by vertical lines on $3-80 \mathrm{keV}$ pulse profile in Figure 3. The phase intervals considered are 0.00-0.18 (phase-1), 0.18-0.43 (phase-2), 0.43-0.70 (phase-3) and 0.70-1.00 (phase-4). The ratio of spectral counts plotted against energy with respect to Crab was fitted with a polynomial of degree 3 along with combination of Gaussians to define corresponding features in the ratio curve. The first phase shows two features around $10 \mathrm{keV}$ and $20 \mathrm{keV}$ and an insignificant feature around $38 \mathrm{keV}$. The feature around $38 \mathrm{keV}$ is more prominent during other phases as well as in the phase averaged case, while the lower two features are not significant during the last two phases. This is borne out by the results of fitting the spectrum. The variation in properties of features with phase effectively rule out instrumental effects or uncertainties in instrumental response as these effects would be independent of phase.

We then derived X-ray spectrum covering a total energy band from $0.8-70 \mathrm{keV}$ with AstroSat, combining SXT and LAXPC20 spectral data. The combined spectrum was fitted with two different models. The first (Model-1) is an absorbed Fermi-Dirac cutoff model, FDCUT (Tanaka 1986) combined with a black body (bbody), a Gaussian for an iron emission line and 3 Gaussian absorption features (gabs) which were introduced to account for presence of cyclotron absorption features in the spectrum to improve the spectral fit. We also tried an alternative model (Model2) an absorbed CompTT model (Titarchuk 1994) combined with an iron emission line and 3 absorption features as described above for comparison and measurement of the centroid energy of the cyclotron absorption features. The spectral parameters derived from these two models are tabulated in Table 2 for phase-averaged spectrum, while Table 3 shows the results of fitting the second model to phase-resolved spectra. Spectra along with the fitted models are shown for phase-averaged and phase-resolved spectra in Figures 7 and 8, respectively. The Figure 9 shows phase dependent variations in spectral residues indicating relative intensities of 3 absorption features individually when the optical depth of corresponding line energy is made zero. In addition, the residue of the fitted model is also presented when all the 3 gabs components, associated with cyclotron resonance scattering features, were removed. This depicts phase dependent variations of relative intensities of cyclotron absorption features for 4 different phases. The difference in $\chi^{2}$ values without the 3 gabs components were established for all the 4 phases and given in the Table 3 . The difference in $\chi^{2}$ values clearly showed overall significance of presence of cyclotron absorption features in the spectrum. A systematic error of $2.5 \%$ was added to account for uncertainties in the response of the instruments. Relative difference in the normalization between instruments was accounted by introducing a constant multiplicative factors for the two instruments and by fixing it for LAXPC20 at 1.0 during the fit. Thus the normalization factor of SXT was found to be 0.31 when a circular field of view of a diameter of $40 \mathrm{arc}$ minutes was selected. This is due to a fixed offset between the pointing axis of the two instruments. The region of interest of SXT, however, was restricted to 12 arc-minutes in diameter to accumulate events mainly from region where source photons dominate, to allow better constrain in the lower energy region of the overall spectrum. With this restricted region of interest, the normalization was found to be 0.18 for SXT with respect to the LAXPC20 frozen at unity. Both the spectral models offered a reasonably good fit to the spectral data and measurements of respective energy of cyclotron absorption features are found to be consistent within their error limits. Considering the observed flux of $4.3 \times 10^{-9} \mathrm{ergs} \mathrm{cm}^{-2} \mathrm{~s}^{-1}$ in the $3-80 \mathrm{keV}$ energy band and 
GRO J2058+42 OUtburSt IN 2019 with AstroSAT

Table 2. Spectral parameters for phase averaged spectrum

\begin{tabular}{|c|c|c|c|}
\hline Parameter & Units & Model 1 (FDCUT) & Model 2 (CompTT) \\
\hline Phabs(nH) & $10^{22} \mathrm{~cm}^{-2}$ & $0.869_{-0.046}^{+0.045}$ & $0.650_{-0.046}^{+0.048}$ \\
\hline $\operatorname{bbody}(\mathrm{kT})$ & $\mathrm{keV}$ & $0.83_{-0.05}^{+0.04}$ & - \\
\hline bbody(Norm) & ph $\mathrm{keV}^{-1} \mathrm{~cm}^{-2} \mathrm{~s}^{-1}$ & $0.0036_{-0.0007}^{+0.0008}$ & - \\
\hline power-law(PI) & - & $1.04_{-0.03}^{+0.03}$ & - \\
\hline$E_{\text {cut }}$ & $\mathrm{keV}$ & $34.6_{-2.1}^{+1.2}$ & - \\
\hline$E_{\text {fold }}$ & $\mathrm{keV}$ & $10.95_{-0.43}^{+0.27}$ & - \\
\hline power-law(Norm) & $\begin{array}{c}\text { ph } \mathrm{keV}^{-1} \mathrm{~cm}^{-2} \mathrm{~s}^{-1} \\
\text { at } 1 \mathrm{keV}\end{array}$ & $0.10_{-0.01}^{+0.01}$ & - \\
\hline CompTT(Redshift) & - & - & 0 (fixed) \\
\hline CompTT(T0) & $\mathrm{keV}$ & - & $0.51_{-0.03}^{+0.02}$ \\
\hline $\operatorname{CompTT}(\mathrm{kT})$ & $\mathrm{keV}$ & - & $8.22_{-0.10}^{+0.10}$ \\
\hline CompTT(tau) & - & - & $5.21_{-0.12}^{+0.12}$ \\
\hline CompTT(approx) & - & - & 0.2 (fixed) \\
\hline CompTT(Norm) & - & - & $0.051_{-0.002}^{+0.003}$ \\
\hline gauss(Line E) & $\mathrm{keV}$ & 6.5 (fixed) & 6.5 (fixed) \\
\hline gauss(Sigma) & $\mathrm{keV}$ & 0.24 (fixed) & 0.24 (fixed) \\
\hline gauss(Norm) & $p h \mathrm{~cm}^{-2} \mathrm{~s}^{-1}$ & $1.8 \times 10^{-4}$ & $2.3 \times 10^{-4}$ \\
\hline gabs(Line E1) & $\mathrm{keV}$ & $10.95_{-0.81}^{+0.74}$ & $10.81_{-0.49}^{+0.48}$ \\
\hline gabs(Sigma) & $\mathrm{keV}$ & $3.74_{-0.53}^{+0.72}$ & $3.20_{-0.32}^{+0.39}$ \\
\hline gabs(Strength) & - & $1.13_{-0.29}^{+0.53}$ & $1.56_{-0.29}^{+0.28}$ \\
\hline gabs(Line E2) & $\mathrm{keV}$ & $21.71_{-0.83}^{+0.89}$ & $20.84_{-0.55}^{+0.29}$ \\
\hline gabs(Sigma) & $\mathrm{keV}$ & $3.61_{-1.32}^{+1.28}$ & $3.69_{-0.40}^{+0.47}$ \\
\hline gabs(Strength) & - & $0.90_{-0.44}^{+0.48}$ & $1.42_{-0.29}^{+0.27}$ \\
\hline gabs(Line E3) & $\mathrm{keV}$ & $38.73_{-0.81}^{+0.44}$ & $38.38_{-1.08}^{+1.14}$ \\
\hline gabs(Sigma) & $\mathrm{keV}$ & $2.64_{-1.33}^{+1.73}$ & $2.56_{-0.77}^{+1.12}$ \\
\hline gabs(Strength) & - & $1.02_{-0.33}^{+0.55}$ & $0.96_{-0.20}^{+0.21}$ \\
\hline Flux $(3-80 \mathrm{keV})$ & $\mathrm{erg} \mathrm{cm}^{-2} \mathrm{~s}^{-1}$ & $4.33 \times 10^{-9}$ & $4.33 \times 10^{-9}$ \\
\hline$\chi^{2}$ & - & 879.79 & 877.36 \\
\hline$\chi_{\text {red }}^{2}($ dof $)$ & - & $1.264(696)$ & $1.257(698)$ \\
\hline \multicolumn{4}{|c|}{ Significance of cyclotron absorption features from F-TEST: } \\
\hline E1 FAP & - & $1.68 \times 10^{-2}$ & $1.79 \times 10^{-4}$ \\
\hline significance $(\sigma)$ & - & 2.39 & 3.75 \\
\hline E2 FAP & - & $2.57 \times 10^{-4}$ & $1.72 \times 10^{-4}$ \\
\hline significance $(\sigma)$ & - & 3.66 & 3.76 \\
\hline E3 FAP & - & $1.37 \times 10^{-7}$ & $1.55 \times 10^{-7}$ \\
\hline significance $(\sigma)$ & - & 5.27 & 5.25 \\
\hline \multicolumn{4}{|c|}{ Significance of cyclotron absorption features from NONE-ZERO LINE DEPTH: } \\
\hline E1 FAP & - & $1.19 \times 10^{-3}$ & $3.14 \times 10^{-6}$ \\
\hline significance $(\sigma)$ & - & 3.24 & 4.66 \\
\hline E2 FAP & - & $4.57 \times 10^{-6}$ & $5.93 \times 10^{-6}$ \\
\hline significance $(\sigma)$ & - & 4.49 & 4.36 \\
\hline E3 FAP & - & $5.89 \times 10^{-13}$ & $2.27 \times 10^{-10}$ \\
\hline significance $(\sigma)$ & - & 7.20 & 6.34 \\
\hline
\end{tabular}

Note-Errors with $90 \%$ confidence range for each parameter. False alarm probability (FAP). 
Table 3. Spectral parameters for phase resolved spectra

\begin{tabular}{|c|c|c|c|c|c|}
\hline Parameter & Units & Phase 1 & Phase 2 & Phase 3 & Phase 4 \\
\hline $\operatorname{Phabs}(\mathrm{nH})$ & $10^{22} \mathrm{~cm}^{-2}$ & $0.83_{-0.11}^{+0.11}$ & $0.81_{-0.12}^{+0.19}$ & $0.65_{-0.08}^{+0.10}$ & $0.64_{-0.08}^{+0.09}$ \\
\hline CompTT(Redshift) & - & 0 (fixed) & 0 (fixed) & 0 (fixed) & 0 (fixed) \\
\hline CompTT(T0) & $\mathrm{keV}$ & $0.41_{-0.05}^{+0.05}$ & $0.42_{-0.11}^{+0.06}$ & $0.53_{-0.05}^{+0.04}$ & $0.47_{-0.04}^{+0.04}$ \\
\hline $\operatorname{CompTT}(\mathrm{kT})$ & $\mathrm{keV}$ & $10.03_{-0.22}^{+0.22}$ & $8.56_{-0.11}^{+0.10}$ & $8.45_{-0.26}^{+0.15}$ & $8.36_{-0.18}^{+0.15}$ \\
\hline CompTT(tau) & - & $4.60_{-0.16}^{+0.17}$ & $6.17_{-0.15}^{+0.16}$ & $4.91_{-0.13}^{+0.27}$ & $4.99_{-0.23}^{+0.11}$ \\
\hline CompTT(approx) & - & 0.2 (fixed) & 0.2 (fixed) & 0.2 (fixed) & 0.2 (fixed) \\
\hline CompTT(Norm) & - & $0.037_{-0.003}^{+0.003}$ & $0.055_{-0.003}^{+0.006}$ & $0.047_{-0.002}^{+0.003}$ & $0.042_{-0.002}^{+0.007}$ \\
\hline gauss(Line E) & $\mathrm{keV}$ & 6.5 (fixed) & 6.5 (fixed) & 6.5 (fixed) & 6.5 (fixed) \\
\hline gauss(Sigma) & $\mathrm{keV}$ & 0.24 (fixed) & 0.24 (fixed) & 0.24 (fixed) & 0.24 (fixed) \\
\hline gauss(Norm) & ph $\mathrm{cm}^{-2} \mathrm{~s}^{-1}$ & $2.10 \times 10^{-4}$ & $2.20 \times 10^{-4}$ & $2.88 \times 10^{-3}$ & $2.88 \times 10^{-3}$ \\
\hline $\operatorname{gabs}($ Line E1) & $\mathrm{keV}$ & $10.26_{-0.52}^{+0.51}$ & $13.17_{-0.99}^{+1.18}$ & $12.44_{-1.18}^{+1.55}$ & $12.05_{-0.89}^{+1.00}$ \\
\hline gabs(Sigma) & $\mathrm{keV}$ & $3.59_{-0.48}^{+0.57}$ & $2.02_{-1.01}^{+1.30}$ & $1.49_{-0.84}^{+1.72}$ & $1.28_{-0.75}^{+1.26}$ \\
\hline gabs(Strength) & - & $2.14_{-0.52}^{+0.58}$ & $0.47_{-0.26}^{+0.20}$ & $0.20_{-0.12}^{+0.26}$ & $0.19_{-0.13}^{+0.11}$ \\
\hline gabs(Line E2) & $\mathrm{keV}$ & $19.72_{-0.40}^{+0.39}$ & $22.20_{-0.78}^{+0.70}$ & $22.68_{-1.15}^{+1.13}$ & $22.11_{-1.33}^{+1.27}$ \\
\hline gabs(Sigma) & $\mathrm{keV}$ & $3.66_{-0.53}^{+0.58}$ & $3.68_{-0.50}^{+0.61}$ & $2.15_{-0.89}^{+1.41}$ & $1.89_{-1.21}^{+1.81}$ \\
\hline gabs(Strength) & - & $2.21_{-0.44}^{+0.57}$ & $1.21_{-0.46}^{+0.60}$ & $0.37_{-0.17}^{+0.62}$ & $0.27_{-0.16}^{+0.17}$ \\
\hline gabs(Line E3) & $\mathrm{keV}$ & $38.38($ fixed $)$ & $40.07_{-1.11}^{+1.15}$ & $38.54_{-0.71}^{+0.70}$ & $41.77_{-1.90}^{+1.35}$ \\
\hline gabs(Sigma) & $\mathrm{keV}$ & $2.2($ fixed $)$ & $4.60_{-0.73}^{+0.87}$ & $2.41_{-1.08}^{+1.11}$ & $5.33_{-1.29}^{+1.45}$ \\
\hline gabs(Strength) & - & $0.059_{-0.059}^{+0.21}$ & $1.69_{-0.36}^{+0.35}$ & $1.15_{-0.23}^{+0.24}$ & $2.39_{-0.75}^{+0.98}$ \\
\hline$\chi^{2}$ & - & 663.48 & 528.95 & 526.29 & 625.61 \\
\hline$\chi_{r e d}^{2}($ dof $)$ & - & $1.211(548)$ & $0.969(546)$ & $0.966(545)$ & $1.148(545)$ \\
\hline$\chi_{\text {diff }}^{2}($ with 3 gabs removed $)$ & - & 224.0 & 68.76 & 66.54 & 62.79 \\
\hline FAP & - & $6.08 \times 10^{-30}$ & $5.42 \times 10^{-11}$ & $1.24 \times 10^{-10}$ & $3.67 \times 10^{-8}$ \\
\hline significance $(\sigma)$ & - & 11.37 & 6.55 & 6.43 & 5.51 \\
\hline \multicolumn{6}{|c|}{ Significance test of cyclotron line using F-TEST: } \\
\hline E1 FAP & - & $9.67 \times 10^{-13}$ & $4.13 \times 10^{-4}$ & $9.29 \times 10^{-2}$ & $9.83 \times 10^{-3}$ \\
\hline significance $(\sigma)$ & - & 7.14 & 3.53 & 1.68 & 2.58 \\
\hline E2 FAP & - & $2.22 \times 10^{-7}$ & $1.58 \times 10^{-6}$ & $2.51 \times 10^{-3}$ & $1.02 \times 10^{-1}$ \\
\hline significance $(\sigma)$ & - & 5.18 & 4.80 & 3.02 & 1.64 \\
\hline E3 FAP & - & $9.9 \times 10^{-1}$ & $7.26 \times 10^{-13}$ & $1.56 \times 10^{-9}$ & $6.11 \times 10^{-9}$ \\
\hline significance $(\sigma)$ & - & 0.01 & 7.17 & 6.04 & 5.81 \\
\hline \multicolumn{6}{|c|}{ Significance test of cyclotron line using NON-ZERO LINE DEPTH: } \\
\hline E1 FAP & - & $4.05 \times 10^{-17}$ & $1.34 \times 10^{-4}$ & $5.13 \times 10^{-2}$ & $3.81 \times 10^{-3}$ \\
\hline significance $(\sigma)$ & - & 8.41 & 3.82 & 1.95 & 2.89 \\
\hline E2 FAP & - & $1.76 \times 10^{-9}$ & $3.89 \times 10^{-7}$ & $1.24 \times 10^{-3}$ & $2.27 \times 10^{-2}$ \\
\hline significance $(\sigma)$ & - & 5.73 & 4.96 & 3.23 & 2.21 \\
\hline E3 FAP & - & $9.26 \times 10^{-1}$ & $1.38 \times 10^{-13}$ & $5.70 \times 10^{-14}$ & $3.01 \times 10^{-11}$ \\
\hline significance $(\sigma)$ & - & 0.09 & 7.40 & 7.08 & 6.65 \\
\hline
\end{tabular}

Note-Errors with $90 \%$ confidence range for each parameter. False alarm probability (FAP) 

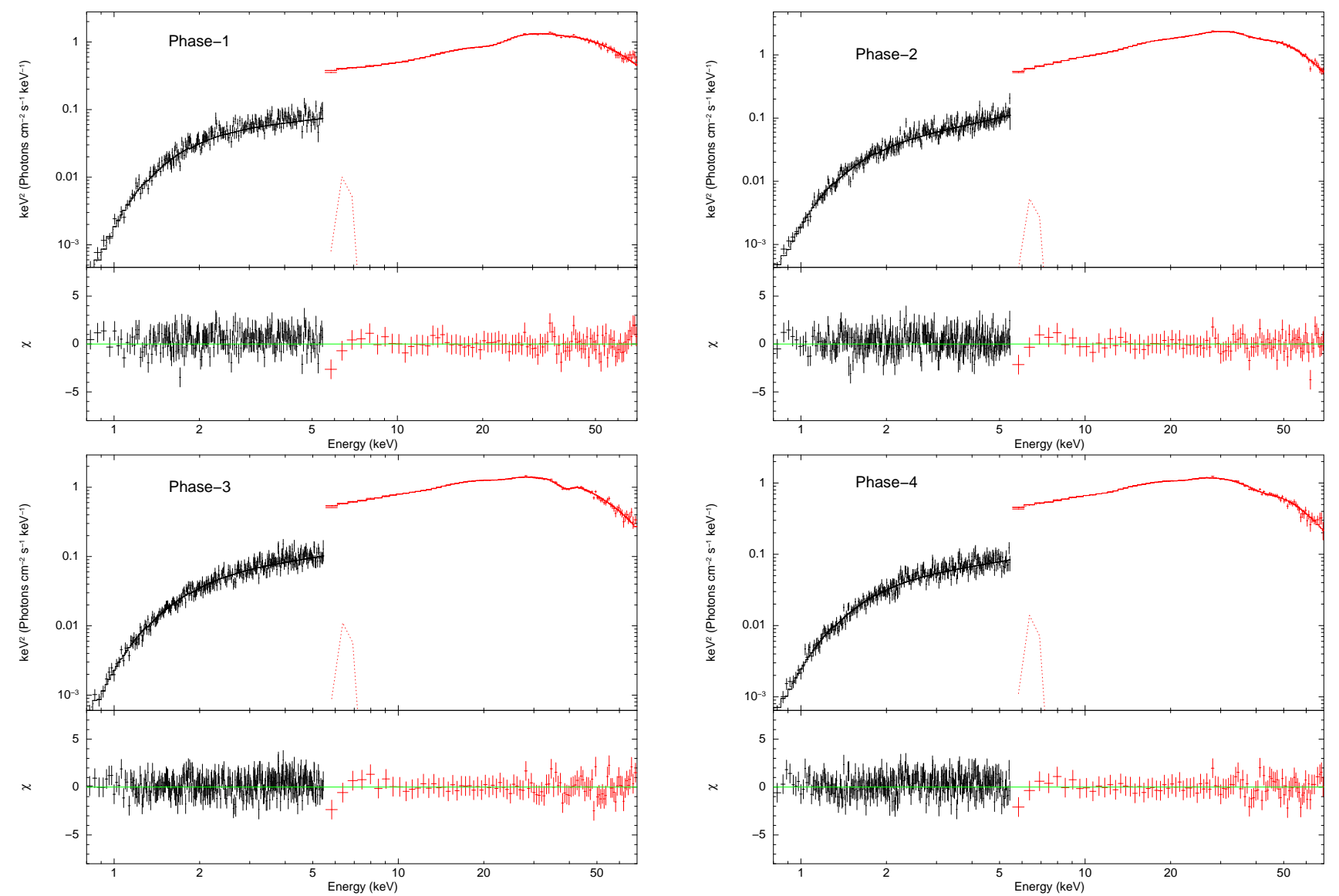

Figure 8. Phase resolved spectra fitted with combined model; an absorbed CompTT with a Gaussian emission line and 3 Gaussian absorptions lines. The graph below shows the residue to the fit in units of chi.

using source distance of $9 \mathrm{kpc}$ (Reig et al. 2005), the X-ray luminosity is determined to be $4.2 \times 10^{37} \mathrm{ergs} \mathrm{s}^{-1}$ during AstroSat observation.

To estimate the statistical significance of the cyclotron absorption features which were detected, we initially tried first two approaches described by Bhalerao et al. (2015) and then cross verified these using Monte Carlo technique as a re-verification for a few cases, as described below.

1. The first approach was to use the F-test, which uses the reduction in $\chi^{2}$ value when the three parameters defining the cyclotron absorption feature using gabs (multiplicative model) were included. Based on the improvement in $\chi^{2}$ value by adding the feature, one could estimate its significance and false alarm probability. However, this is to specifically mention that the F-test used here is additive and has certain limitations for multiplicative models such as gabs-model (Orlandini et al. 2012; Protassov et al. 2002). This test was applied for an initial assessment and for rough estimates of the significance of 3 individual additional components, defined by gabs-model. In all cases, the significance of each feature was calculated independently and we have calculated the probability of the signal being false and converted that to the significance in terms of equivalent value of $\sigma$ in the normal distribution. The estimated significance by this F-test for the absorption features in the spectrum are tabulated for phase averaged and 4 different pulse phases in Tables 2 and 3, respectively.

2. In the second approach, we kept the centroid energy fixed for a cyclotron absorption feature and stepped through a grid of values of line-depth and width in steps to study the change in $\chi^{2}$ as a function of these two parameters, using the XSPEC steppar command. The change in the minimum $\chi^{2}$ value corresponding to zero line-depth was noted. Thus, using the minimum difference in $\chi^{2}$ required to get zero line-depth, we could calculate the false alarm probability and the significance of the line. The results are shown in Table 2 for phase averaged spectrum and in Table 3 for four different pulse-phases of the pulsar. These estimates of significance of cyclotron absorption features are consistent with the estimates using option (1). 

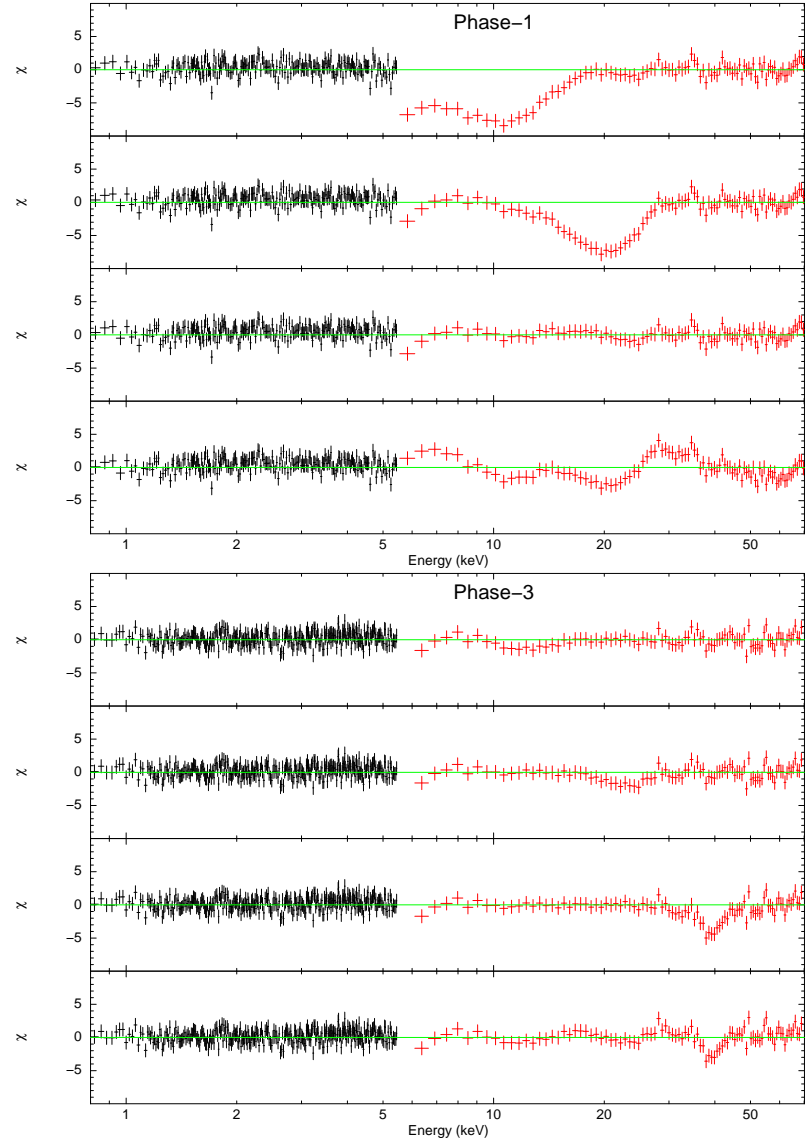
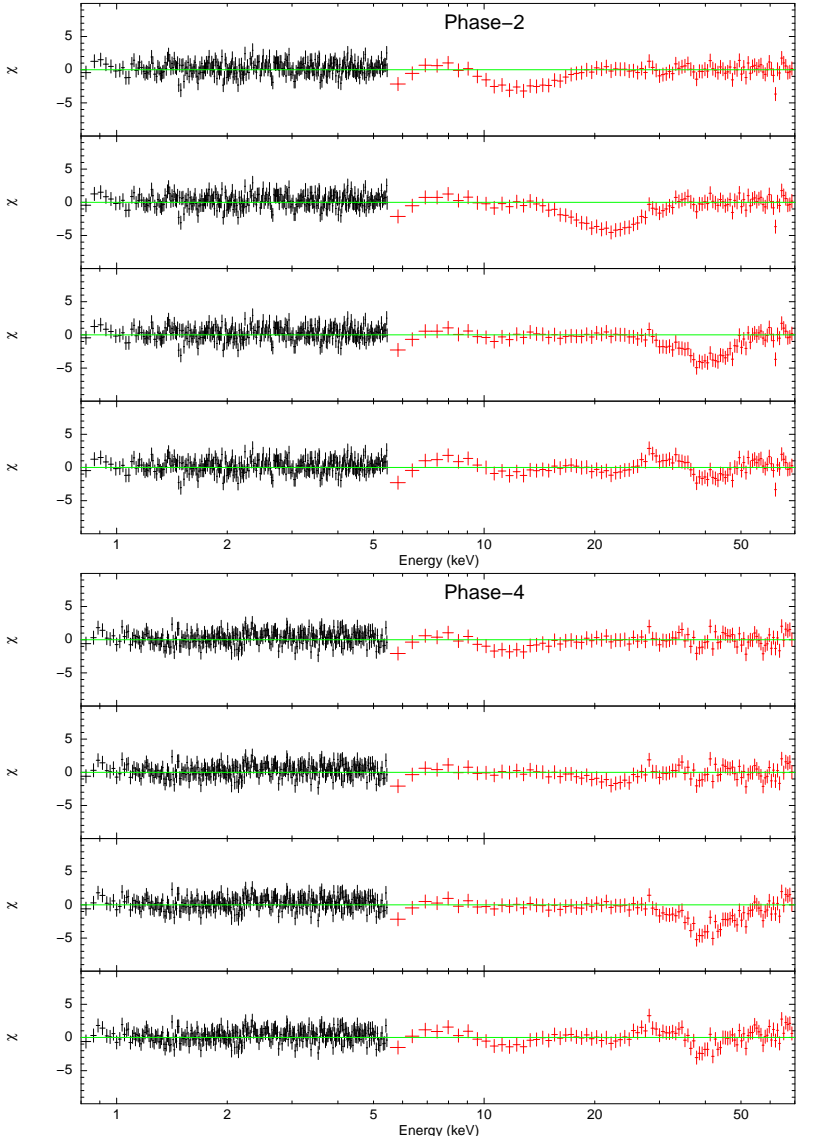

Figure 9. Phase resolved spectra residues showing relative intensities of 3 absorption lines (top 3 panels of each figure) when individual optical depth is made zero and followed by fitted residue when all the three absorption features were removed from the model (bottom panel of each figure) for all the 4 different phases. The combined spectrum was fitted with Model 2.
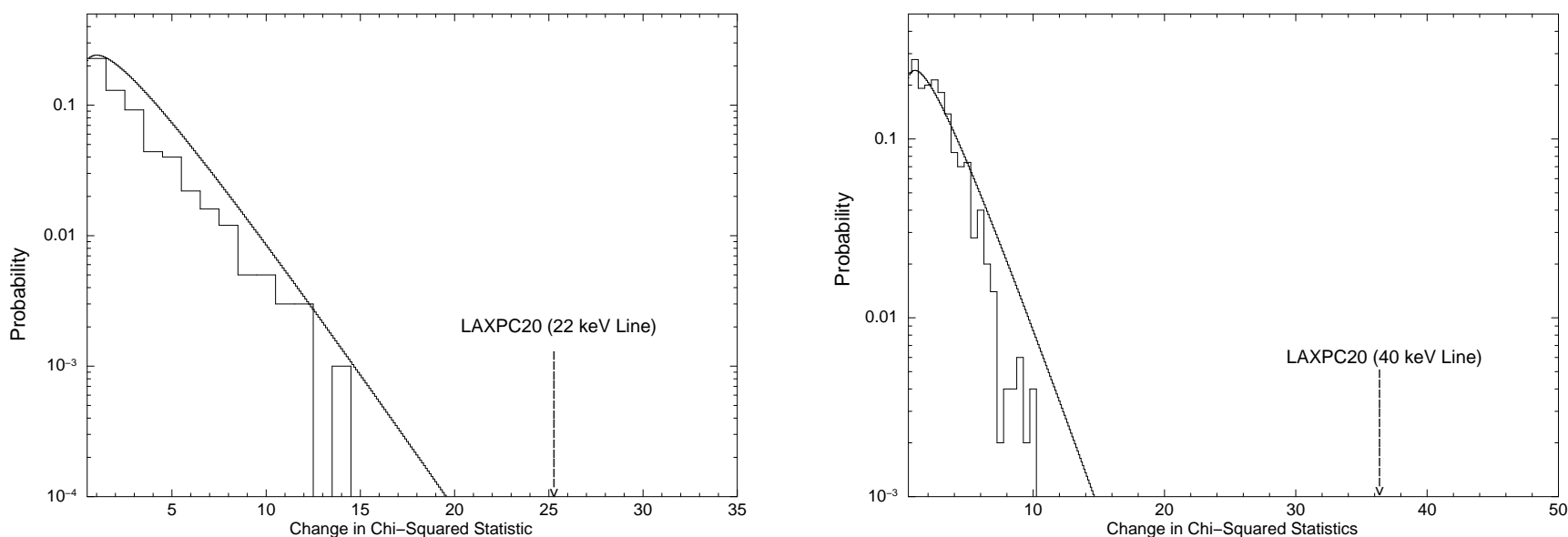

Figure 10. Results of $\Delta \chi^{2}$ distribution derived from Monte Carlo simulations of spectra for LAXPC20 for testing significance of cyclotron absorption features corresponding to lines at $\sim 22 \mathrm{keV}$ (left panel) and $\sim 40 \mathrm{keV}$ (right panel) are shown for pulse phase-2. The observed values of $\Delta \chi^{2}$ corresponding to above two lines are shown by vertical arrows.

3. We also assessed the significance of cyclotron absorption features independently using Monte Carlo simulation. This technique has been used to establish significance of cyclotron absorption features in the spectrum for many sources (Bellm et al. 2014; Bhalerao et al. 2015; Bodaghee et al. 2016). We used LAXPC20 data fitted with the CompTT continuum model along with Fe-line and 3 Gaussian absorption features which were introduced 
to model observed cyclotron absorption features. Only LAXPC20 data were considered to improve on the speed of simulation and convergence to the fit. We fixed the Hydrogen column density $\left(N_{H}=0.81 \times 10^{22}\right.$ $\mathrm{cm}^{-2}$ ) as derived from fit to the combined SXT and LAXPC20 spectral data. We then used XSPEC script 'simftest' for simulating 1000 spectra. The script does the fit with and without cyclotron absorption feature for each of the simulated data. From these simulations, one can estimate the change in $\chi^{2}$ between a model with and without the cyclotron absorption feature and find the distribution of $\Delta \chi^{2}$ values. These simulations were done to re-confirm the significance results for Phase- 2 spectrum, corresponding to its $\sim 22 \mathrm{keV}$ and $\sim 40 \mathrm{keV}$ cyclotron absorption features, independently. The change in chi-squared $\left(\Delta \chi^{2}\right)$ distribution were plotted. Since the cyclotron absorption feature is defined by three free parameters, we expect the simulated $\Delta \chi^{2}$ distribution to follow a $\chi^{2}$ distribution with three degrees of freedom. The observed distribution was found to be consistent with the $\chi^{2}$ distribution as can be seen in the Figure 10. In these two cases maximum values of $\Delta \chi^{2}$ obtained in simulation was 14 (22 keV line) and 10 (40 keV line) which are much lower than the observed values of 25.5 (22 keV line) and 36.7 (40 keV line) in the LAXPC20 data. By extrapolating the distribution we estimate that a very large number of simulations would be required to get observed value of $\Delta \chi^{2}$ in one of them by chance. This could be $10^{5}-10^{6}$ simulations required for $\Delta \chi^{2} \approx 26$ and $10^{8}-10^{9}$ for $\Delta \chi^{2} \approx 37$. Therefore, it is not feasible to perform these many simulations to achieve desired values. The significance of individual features for LAXPC20 data for phase-2, corresponding to these values are $4.38 \sigma\left(\mathrm{FAP}=1.2 \times 10^{-5}\right)$ for $22 \mathrm{keV}$ line and $5.4 \sigma$ $\left(\mathrm{FAP}=5.32 \times 10^{-8}\right)$ for $40 \mathrm{keV}$ line. These simulations therefore confirmed presence of these features with high significance in LAXPC20 spectrum which detected cyclotron absorption features from the source in its sensitive energy band. It thus also confirms that the significance established from the first two methods as shown in the Table 3 are consistent with the results obtained by the Monte Carlo simulation technique as shown here as a test case. We have tried a few more cases and in all of these the significance is consistent with those obtained using options (1) and (2).

Our results for phase-1 are in agreement with Molkov et al. (2019), whereby the first two features around 10 keV and $20 \mathrm{keV}$ were detected with high significance and no other higher energy features were detected. The mean energy, width and depth of the features estimated by us is also consistent with those by Molkov et al. (2019). Additionally, we detected absorption feature around 38-40 keV for the rest of the 3 phases with high significance. However, we did not detect presence of any cyclotron absorption feature around $30 \mathrm{keV}$ as reported by Molkov et al. (2019). This was verified using phase-1 spectrum, where a third gabs model was introduced with frozen values of its energy and width as reported by (Molkov et al. 2019) and its optical depth was allowed to vary. The additional gabs-model fitted to the spectral data could not detect any significant optical depth corresponding to the energy $\sim 30 \mathrm{keV}$. The individual spectrum, Figures $4 \& 5$ of Molkov et al. (2019), do not appear to show significant feature around 30 keV. The feature seen around 38-40 keV in LAXPC spectrum, could possibly be either the third harmonic (if the primary absorption feature is around $12 \mathrm{keV}$ ) or the fourth harmonic (if the primary feature is around $10 \mathrm{keV}$ ). In the latter case, we may have missed the third harmonic as it could possibly be very weak and hence not detectable. Even higher harmonics could be present, but due to lower sensitivity at high energies it is difficult to say anything definitely about them. We thus detected a cyclotron absorption feature around $38-40 \mathrm{keV}$ in the other 3 phases, which is contrary to the results of Molkov et al. (2019). This detection was possible due to relatively higher effective area of the LAXPC20 (Antia et al. 2017) by more than an order of magnitude around 38-40 keV compared to FM-modules of the NuSTAR (Harison et al. 2013; Brejnholt et al. 2012). This is evident from their Figure 4 and 5, (Molkov et al. 2019), where errors are relatively large around $38 \mathrm{keV}$ and hence it is likely that $38 \mathrm{keV}$ feature was not detected by the NuSTAR. We detected $38 \mathrm{keV}$ absorption feature with high significance as reported in Table-2, even in the phase averaged spectrum. We also noticed that the spectral ratio with respect to Crab spectrum (Figure 6) clearly showed depression around 38-40 keV, confirming presence of this absorption feature in the spectrum and the variation in its shape with phase. This rules out the possibility of occurrence of absorption feature due to inaccuracy of instrument response or the modeled background.

\section{DISCUSSION}

\subsection{Power Spectrum and $Q P O$}

Many accretion powered pulsars show milli-Hertz quasi periodic oscillations (QPOs). X-ray pulsars with high mass companions such as X Per (Takeshima 1998), 4U 1907+09 (In’t Zand et al. 1998; Mukerjee et al. 2001), XTE 
J1858+034 (Paul \& Rao 1998), A 0535+26 (Finger et al. 1996), V 0332+53 (Takeshima 1994) and X 0115+63 (Soong \& Swank 1989) showed QPOs with their respective peak oscillation frequencies in the range 50-110 mHz. QPOs in X-ray pulsars offer an important diagnostic tool to probe; accretion flows in these binaries, conditions in the inner region of its accretion disk, properties of accretion torques exerted on its neutron stars and disk-magnetospheric coupling and their interaction (Ghosh 1996, 1998).

Now let us consider two main models and their applicability to high mass X-ray binary GRO J2058+42. These are namely: Keplerian frequency and Beat frequency model (van der Klis 2000). The Keplerian frequency model, where QPOs are produced due to inhomogeneities at the inner edge of the Keplerian disk, that modulate the X-ray flux at Keplerian frequency, expressed as $\nu_{\mathrm{QPO}}=\nu_{k}$ (van der Klis et al. 1987). Whereas in the case of Beat frequency model, the accretion flow on to the neutron star is modulated at the Beat frequency between Keplerian frequency at the inner edge of the accretion disk and the neutron star spin frequency, $\nu_{\mathrm{QPO}}=\nu_{k}-\nu_{s}$ (Alpar \& Shaham 1985). In the case of GRO J2058+42, the detected QPO frequency $\nu_{Q P O}=9.0 \times 10^{-2} \mathrm{~Hz}$ is much higher than the spin frequency of its neutron star, $5.15 \times 10^{-3} \mathrm{~Hz}$. It is, therefore, not possible to differentiate between a Beat frequency and a Keplerian frequency model in the case of GRO J2058+42.

We can obtain radius of the inner edge of the accretion disk $r_{\mathrm{QPO}}$ using expression for Keplerian orbital motion,

$$
r_{\mathrm{QPO}}=\left(\frac{G M}{4 \pi^{2}}\right)^{1 / 3} \nu_{k}^{-2 / 3} \approx 8.0 \times 10^{8} \quad \mathrm{~cm}
$$

where $M$ is $1.4 M_{\odot}$ for a neutron star and $G$ is the gravitational constant and $\nu_{k}$ its Keplerian frequency.

Alternatively, using mass accretion rate and strength of magnetic field of neutron star one can also derive radius of the inner edge of the Keplerian disk, which is equivalent to the magnetospheric radius $r_{m}$, of the neutron star as expressed by the equation 6.18 , page 158 of Frank et al. (2002)

$$
r_{m} \simeq 5.2 \mu_{30}^{4 / 7} \dot{M}_{16}^{-2 / 7} m_{x}{ }^{-1 / 7} \times 10^{8} \mathrm{~cm}=2.4 \times 10^{8} \mathrm{~cm},
$$

where $m_{x}=M_{x} / M_{\odot}=1.4, \mu_{30}$ is magnetic moment of the neutron star expressed in units of $10^{30} \mathrm{G} \mathrm{cm}^{3}$ and $\dot{M}_{16}$ is its mass accretion rate expressed in units of $10^{16} \mathrm{~g} \mathrm{~s}^{-1}$. These are derived from observed values of neutron star magnetic field and source luminosity respectively as detected by AstroSat observation.

The co-rotation radius $r_{c o}$, of an X-ray pulsar can be defined where the spin angular velocity of neutron star is equal to the Keplerian angular velocity of matter. It can be derived by equating the Keplerian velocity to the co-rotating Keplerian velocity,

$$
r_{c o}=1.7 \times 10^{8} P^{2 / 3}\left(\frac{M}{1.4 M_{\odot}}\right)^{1 / 3} \mathrm{~cm},
$$

where $P$ is the spin period of the neutron star. Using estimated pulse period from AstroSat observation and assuming a neutron star mass of $M=1.4 M_{\odot}$, one can obtain the co-rotation radius $r_{c o}$ for GRO J2058+42 as $5.7 \times 10^{9} \mathrm{~cm}$ (Equation-6). It is therefore evident that the disk radius $r_{\mathrm{QPO}}$ is about an order of magnitude smaller than the corotation radius $r_{c o}$. It suggests, therefore, that formation of such a transient disk is possible between magnetosphere and co-rotation radius of the neutron star. Estimated values of the radius of the inner accretion disk derived by Keplerian orbital motion is $8.0 \times 10^{8} \mathrm{~cm}$ (Equation-4) and using the accretion torque theory, it is determined to be $2.4 \times 10^{8}$ $\mathrm{cm}$ (Equation-5). These values are comparable considering the level of approximations involved, and uncertainties in mass, radius and distance of the source as well as in the geometry of the magnetic field. It favors however, formation of a transient accretion disk around the neutron star, which could possibly explain the cause of observed oscillations at $0.090 \mathrm{~Hz}$ in the X-ray flux due to either Beat frequency or Keplerian frequency modulations as discussed above. Such properties are quite recurrent in many cases of Be-binary pulsars as mentioned above and also observed in this case, for the first time by LAXPC on-board AstroSat. The neutron star magnetospheric radius established for some cases (Nespoli \& Reig 2011; Devasia et al. 2011) using measured strength of magnetic field, source distance and its luminosity, assuming the canonical mass and radius of a typical neutron star (Equation-5) was found to be very close to inner radius of the accretion disk, as determined from its observed QPO frequency (Equation-4) for some sources. Hence, for such cases, it was possible to establish consistency with spectroscopic measured value of their magnetic field strength. The same appears to be true for GRO J2058+42 under uncertainty involved in measured parameters and standard assumptions. 
Formation of a transient disk may supply necessary spin-up torque to the neutron star, if the disk rotates in the same direction as the pulsar spin. The expected torque $\left(N_{\text {char }}\right)$ on to the neutron star due to transfer of angular momentum of the accreted mass from such a transient disk can be calculated using the expression below, assuming, transfer of angular momentum of all accreted mass from such a disk with a radius $r_{Q P O}$ to its neutron star (In't Zand et al. 1998; Mukerjee et al. 2001)

$$
N_{\text {char }}=\eta \dot{M}\left(G M r_{Q P O}\right)^{1 / 2} \text {, }
$$

where $\dot{M}$ is the mass accretion rate and $\eta$ is the duty cycle for the applied torque.

If we assume that all the potential energy of the accreted mass liberated during the outburst is transformed into radiation then the luminosity can be expressed as,

$$
L_{37}=1.33 \dot{M}_{17}\left(M / M_{\odot}\right) R_{6}{ }^{-1} \operatorname{erg~s}^{-1}
$$

where $L_{37}$ is the luminosity in units of $10^{37} \mathrm{erg} \mathrm{s}^{-1}$ and $R_{6}$ is the radius in units of $10^{6} \mathrm{~cm}$. The mass accretion rate can be calculated from the observed luminosity, which is derived by measurements of flux from spectral model for AstroSat spectrum (Table 2), and known distance of the star. The estimated value of $\dot{M}$ during the AstroSat observation was $2.3 \times 10^{17} \mathrm{~g} \mathrm{~s}^{-1}$. Using these parameters, the value of $N_{\text {char }}$ is found to be $\eta\left(9.0 \times 10^{34}\right) \mathrm{g} \mathrm{cm}^{2} \mathrm{~s}^{-2}$.

The observed torque $N_{0}$ of the pulsar can be expressed in terms of the moment of inertia, $I=10^{45} \mathrm{~g} \mathrm{~cm}^{2}($ for a typical neutron star, having mass as $1.4 M_{\odot}$ and radius of $10 \mathrm{~km}$ ) and $\dot{\nu}$ the observed rate of change of frequency of the neutron star as

$$
N_{0}=2 \pi I \dot{\nu}
$$

Now, if we consider the value of $\eta \approx 1.0$, representing that the transient accretion disk was present and torque was applied for almost the whole duration of observation. Then, by equating the above two torques (Eqs. 7 and 9 ), one can estimate an average spin-up rate of the pulsar as $\dot{\nu}=1.43 \times 10^{-11} \mathrm{~Hz} \mathrm{~s}^{-1}$ or $\dot{P}=-5.39 \times 10^{-7} \mathrm{~s} \mathrm{~s}^{-1}$. This is comparable to the observed value of $\dot{\nu}$ during AstroSat observation.

Considering the value of $\dot{\nu}=1.65 \times 10^{-11} \mathrm{~Hz} \mathrm{~s}^{-1}$ obtained during the AstroSat observation and assuming that the spin-up rate is proportional to the luminosity during the recent outburst we could integrate $\dot{\nu}$ over the entire outburst to calculate the net change in spin frequency or the period. Taking into account the observed period of $194.22 \mathrm{~s}$ during the beginning of AstroSat observation we could calculate that the period decreased from about $195.7 \mathrm{~s}$ before outburst $(\mathrm{MJD}=58550)$ to $193.4 \mathrm{~s}$ after this latest long outburst of 2019 (MJD $=58624$ ). This change in period is comparable to the measured period change from 195.6 to 193.5 by Fermi-GBM during this latest outburst. This is also comparable to the period change during earlier outburst observed by BATSE in 1995. Thus, the formation and presence of such a transient accretion disks around neutron star during the source outburst, could cause significant change in the pulsar period in short duration of 46 days.

\subsection{Pulse Phase-averaged Spectrum}

The X-ray spectrum of the source was first derived by RXTE during the earlier outburst of 1996. The RXTE PCA and HEXTE data were used covering 2.7-25 keV and 11-50 keV respectively (Wilson et al. 2005). The spectral data were fitted with an absorbed thermal bremsstrahlung model (Phabs*bremss) adequately well, particularly at higher intensities during its outburst. The source flux dropped sharply above $20 \mathrm{keV}$ in most cases, therefore, other models such as a power law with a high-energy cutoff, were not used with RXTE data. It was found that the absorption term, $N_{H}$ was nearly constant with its best-fit values in the range $(4.6-5.4) \times 10^{22} \mathrm{~cm}^{-2}$. The temperature, kT, was found to increase with the source intensity, with best-fit values varying from $10.3 \pm 0.5 \mathrm{keV}$ at $2.9 \times 10^{-11} \mathrm{ergs}^{\mathrm{cm}} \mathrm{s}^{-1}$ to $22.2 \pm 0.4 \mathrm{keV}$ at $2.6 \times 10^{-10} \mathrm{ergs} \mathrm{cm}^{-2} \mathrm{~s}^{-1}$ (Wilson et al. 2005). There was, however, no report on any cyclotron absorption feature from RXTE observations. This was missed very likely due to observations at much lower intensity of the source during low intensity outbursts, which was an order of magnitude lower than the latest outburst.

AstroSat data enabled us to derive X-ray spectrum in 0.8-70 keV energy band as shown in Figure 7 . Spectrum was fitted reasonably well using two models. The first model was defined as an absorbed Fermi-Dirac cutoff model along with a black-body, a Fe-emission line and 3 Gaussian absorption lines were introduced to model cyclotron scattering features and its two higher harmonics as observed in the spectrum. The CompTT model was used as the second model in combination with a Fe-emission line and 3 Gaussian absorptions lines as defined in the first model. The CompTT-model is generally used for neutron star based low mass X-ray binaries such as Z-type and atoll sources with relatively lower magnetic field $\left(<10^{12} \mathrm{G}\right)$ of the neutron star (Ferinelli et al. 2008). However, the model could 
also successfully define spectrum of some of the pulsars in Be-binaries for example Cep X-4 (Jaiswal et al. 2015), 4U 1907+09 (Varun et al. 2019) and GRO J2058+42 (Molkov et al. 2019). The derived parameters from the two models are tabulated in Table 2. The first model estimated a black-body temperature of $0.83 \pm 0.04 \mathrm{keV}$ and detected presence of cyclotron resonance scattering feature and its harmonics. The Comptonization model on the other hand enabled us to determine input photon Wien-temperature of $0.52 \pm 0.02 \mathrm{keV}$, the plasma temperature of $8.22 \pm 0.10 \mathrm{keV}$ and plasma optical depth of $5.21 \pm 0.12$ for the phase-averaged spectra. The Wien-temperature, as per CompTT-model, originates away from the neutron star surface and closer to the inner accretion disk i.e., at the outer transition layer, hence Wien-temperature is always found to be relatively lower than the neutron star black-body temperature as it originates close to inner transition layer i.e., near the surface of the neutron star (Ferinelli et al. 2008). As per the CompTT model bulk Comptonization occurs in the innermost part of the transition-layer region, while thermal Comptonization is dominant in the outer transition layer and presumably within some extended region located above the accretion disk. Similar deviations were also observed in the case of Cep X-4 fitted with CompTT-model and black-body combined with FDCUT-model (Table 1 of Jaiswal et al. 2015). However, the centroid energy of cyclotron absorption feature and its detected harmonics are found to be consistent within errors for the two models (Table 2).

Some of the accretion powered X-ray pulsars showed additional features in emission between 10-20 keV energy band and more rarely in absorption between $8-10 \mathrm{keV}$ in their respective residuals when fitted with a variety of continuum models (Coburn et al. 2002). Coburn et al. (2002) have argued that such features may be caused by inadequacies of continuum model rather than cyclotron resonance features. For example, it was observed that a single emission line at around $14 \mathrm{keV}$ can fit two features around 10 and $20 \mathrm{keV}$ for Vela X-1 (Kreykenbohm et al. 2002), Her X-1 (Coburn W. 2001), Cep X-4 (McBride et al. 2007) and an absorption line between 8-10 keV can fit the features for 4 U 1907+09, $4 \mathrm{U}$ 1538-52, 4U 0352+309 as in figure 6 of Coburn et al. (2002). In the case of GRO J2058+42, an introduction of a single Gaussian emission line in this range of energy could not appropriately fit the spectrum. Referring to the results described in Section 3.3 using ratios of spectral counts with respect to Crab spectrum derived for 4 different phases of the pulsar (Figure 6), clearly indicates presence of prominent depressions around $10 \mathrm{keV}$ and $20 \mathrm{keV}$ for phase- 1 in particular, and its presence at other phases as well. Therefore, it confirms the presence of such absorption features in the spectral data associated with a physical origin and not due to any discrepancies of the continuum model as discussed above. Additionally, it also excludes the possibility of any uncertainty in the response matrix of the detector as the response matrix was not used to deconvolve the spectrum to calculate these ratios. Relative significance of these absorption features were subsequently estimated after modeling the data and results are shown in Table 2 for the phase averaged case and in Table 3 for 4 different pulse phases. These observations strongly favor presence and detection of these absorption features in their respective spectra of the pulsar.

The Be-binary pulsar $4 \mathrm{U} 0115+63$ showed interesting features when its measured pulsed fraction was plotted against energy starting from $5 \mathrm{keV}$ onwards. It showed gradual increase in pulsed fraction with energy along with a sharp localized decrease only around $22 \mathrm{keV}$. This localized decrease was attributed to cyclotron resonant scattering at the second Landau level. No such localized decrease was observed for its higher harmonics likely due to low signal to noise ratio. Such decrease was also not observed corresponding to its fundamental line, despite its high count rate, as it could be due to competing effects such as photon spawning and cyclotron emission (Ferrigno et al. 2009). The pulse fractions measured at different energies between 3-80 keV from AstroSat observation of GRO J2058+42 (Figure 4) did not show any localized decrease in its pulse-fraction corresponding to fundamental $(\sim 10 \mathrm{keV})$ or even for its observed higher harmonics $(\sim 20 \mathrm{keV}, \sim 38 \mathrm{keV})$. These could possibly be due to similar reasons mentioned as above for $4 \mathrm{U}$ $0115+63$ for fundamental and higher harmonics or the resultant decrease in pulse fraction is small and it could not be detected within the error limits. This is very similar to another Be-binary V $0332+53$, where no such localized change in pulsed-fraction was observed corresponding to its cyclotron line energies, despite their prominent optical depths (Tsygankov et al. 2010). The pulse fraction for GRO J2058+42, however, showed a gradual increase with energy and then became nearly constant at higher energies above $20 \mathrm{keV}$ as seen in Figure 4.

\subsection{Pulse Phase-resolved Spectra}

Cyclotron resonant scattering features are produced due to interaction of photons with electrons quantized in the Landau states formed in the accretion column in the presence of a strong magnetic field of a neutron star in accreting X-ray binaries (Schwarm et al. 2017). It can also be produced by reflection of X-rays from the atmosphere of the neutron star (Poutanen et al. 2013). These interactions results in absorption features in their spectrum at a particular energy and produce complex shape of the absorption features due to complex scattering cross-sections. The line 
energies and their shapes change depending on the environment of the line-forming region typically located close to the neutron star, strength of magnetic field of the neutron star, nature of the accretion column, source luminosity and pulse-phase of accreting X-ray pulsars (Nishimura 2003, 2005, 2013).

The pulse phase-resolved spectra corresponding to 4 different phases of the accreting pulsar GRO J2058+42 are presented in Fig. 8. AstroSat detected a cyclotron absorption feature and its two harmonics in its pulse phase-resolved spectra, which were identified in the phased-averaged spectrum. Spectral parameters derived from the fitted CompTTmodel for 4 different pulse phases are given in Table 3. It is noticed that the relative strengths and shape of these absorption features change with pulse-phase as shown in Figure 9 for clarity. The line energies are found to vary within the range of (9.7-14.4) keV, (19.3-23.8) keV and (37.3-43.1) keV respectively for the observed cyclotron scattering features and its higher harmonics. Their respective detection significance along with false alarm probability (FAP) with respect to pulse-phases are also given in Table 3. We noticed a higher value of centroid energy of the fundamental cyclotron line energy at 13.17 for phase-2, but it was found to be consistent with phase- 3 and Phase- 4 within its associated larger uncertainty. Overall pulse phase-resolved spectra of GRO J2058+42 showed consistency with respect to cyclotron line energies and continuum except for the phase-1 (Table 3).

The phase averaged spectrum (Figure 7), fitted using CompTT-model for GRO J2058+52 determined the cyclotron fundamental line energy along with the harmonics from AstroSat observation. Interestingly, the observed ratios between its fundamental and the two harmonics are found to cover a range of $1.93 \pm 0.06$ and $3.6 \pm 0.12$ respectively with a $1-\sigma$ error limit. This indicates non-harmonic ratio, observed for higher harmonics of cyclotron resonance scattering feature with respect to its fundamental line energy. However, looking at the results from pulse phase resolved spectra in Table 3, it is clear that the position of the spectral features as well as their strengths showed variation with phase but when compared with phase averaged spectrum it may give misleading results. For example, the mean position of the first feature is different in Phase- 1 as compared to other phases and its strength is highest in Phase- 1 . Thus the phase averaged spectrum is likely to be biased towards the lower value in Phase-1. On the other hand, the third feature around $38 \mathrm{keV}$ is not seen in Phase-1, while it is significant in other phases and the phase averaged spectrum will reflect this value. If we consider the ratios between energies of different features in phase resolved spectra (Table 3), the ratios for the first two features are $1.69 \pm 0.16,1.82 \pm 0.14$ and $1.84 \pm 0.11$ corresponding to phase-2, phase- 3 and phase-4 respectively. Similarly, the ratio between the third and the first feature, ignoring Phase-1 where the third feature is not significant, are $3.04 \pm 0.17,3.10 \pm 0.23$ and $3.47 \pm 0.20$ for the three phases respectively. Within the error bars these ratios are consistent with those expected for harmonics. Hence, there is no evidence for non-harmonic ratios in GRO J2058+42. However, non-harmonic ratios in cyclotron absorption features were observed for some of the pulsars, for example V 0332+53 (Pottschmidt et al. 2005; Kreykenbohm et al. 2005; Nakajima 2010), Her X-1 (Enoto et al. 2008) and Cep X-4 (Vybornov et al. 2017). For some cases, marginal deviation in harmonicity could be explained by employing relativistic approximation of photon-electron scattering (Meszaros, et al. 1992), but for large deviations it could possibly be explained due to influence of non-dipole magnetic field on the line forming region when the field strength increases with height (Nishimura 2005, 2013).

The variation in cyclotron features with pulse phase could be due to superposition of contributions from a number of lines formed at different heights of a line-forming region in a cylindrical accretion column geometry, influenced by a large gradient of its magnetic field. This could result in the observed variation of the centroid energy and shape depending on visibility of accretion column with respect to line of sight depending on system orientation during spin of the neutron star. This could occur either in the accretion column or in the neutron star atmosphere for an anisotropic injection of energy with an emission peak exiting in a particular direction (Nishimura 2015). In such cases, cyclotron scattering features could be observed only for the interval depending on its pulse-phase as seen for pulse phase- 1 for GRO J2058+42. There could be other possibilities where the accretion column itself could partially be eclipsed by the neutron star itself for certain pulse-phases, such that only a portion of the accretion column could be visible to the observer (Mushtukov et al. 2018). This may cause dispersion in magnetic field strength across the visible portion of the accretion column and may cause resultant change in the line-energy and its shape with pulse-phase. This suggest that the observed phase dependent changes in the cyclotron line energy and its shape for GRO J2058+42 during AstroSat observation, could likely be due to changes in the geometry of the line producing region with respect to line of sight during its spin, for a stable luminosity of the source.

We find from Table 3 that photo electric absorption and input soft photon temperature CompTT(T0) for all the 4 phases were almost constant within $90 \%$ error limit. The plasma temperature CompTT(kT) for phase- 1 was, however, found to be relatively higher by $\sim 1.5 \mathrm{keV}$ as compared to an average of the remaining 3 phases at $8.5 \mathrm{keV}$. The 
plasma optical depth for phase- 2 was found to be higher at 6.17 compared to its average value of 4.8 for the other 3 phases. These measurements indicated that for the phase-1, the difference in plasma temperature and possibly local change in configuration of its magnetic field could be responsible for the observed change in its continuum and line parameters as compared to the other 3 phases. The observed ratio of the spectral counts with respect to Crab for phase-1 also clearly showed that there are significant changes in the spectrum relative to other phases (Figure 6). Therefore, the evident differences in its continuum and line parameters indicate that for this narrow phase-1, emission probably comes mainly from a different region and hence emission components are different with respect to the rest of the three phases. For example, the radiation could originate from one column instead of another, from its visible higher column height as opposed to near the stellar surface. The stellar surface area associated with a relatively hotter plasma accumulation may also be suggestive of a scenario where a magnetically more intense area produces a hotter region contributing a substantial variation in the spectral shape due to the interaction of accelerated high energy particles which could up-scatter soft photons giving rise to the Comptonization spectrum with change in its spectral shape. The recent work of (Nishimura 2019) could explain more favorably the observed change in spectral continuum as well as decrease in the centroid energy of the cyclotron lines in general and in particular that of the phase-1. The velocity of bulk motion of in-falling plasma in the cyclotron line forming regions plays a dominant role. Line forming regions are located near the walls of the cylindrical accretion column whereas spectral continuum are formed above and around the accumulated mound close to neutron star surface. Therefore, relative location of these two regions where continuum and cyclotron-lines are formed with respect to a line of sight of the observer, one could see the observed change in the spectral continuum as well as a decrease in centroid energy of the cyclotron-line during certain pulse-phase of the pulsar depending on optimal conditions such as velocity of the bulk motion of the in-falling matter, effect of gravitational bending of the emitted radiation and suitable variation of the local magnetic field (Nishimura 2019). The fundamental centroid energy of the cyclotron absorption feature showed overall variation of $\sim 30 \%$ with pulse-phase measured for GRO J2058+42. There are several other sources, namely Cen X-3 (Burderi et al. 2000; Suchy et al. 2008), Vela X-1 (La Barbera et al. 2003; Kreykenbohm et al. 2002), 4U 0115+63 (Heindl et al. 2004), GX 301-2 (Kreykenbohm et al. 2004; Suchy et al. 2012), and Her X-1 (Voges et al. 1982; Soong et al. 1990; Klochkov et al. 2008), which showed variation in their fundamental energy of about 10-30\% over the pulse-phase. The cyclotron absorption features of GRO J2058+42 clearly showed comparable variation in cyclotron line-energy and shape with variation in its pulse-phase.

\subsection{Determination of the Strength of Magnetic Field of the Pulsar}

The strength of the surface magnetic field B of a neutron star can be obtained using

$$
E_{c} \simeq 11.6 n\left(\frac{1}{1+z}\right)\left(\frac{B}{10^{12} G}\right) \mathrm{keV} .
$$

We assume that the observed cyclotron absorption feature at $E_{c}$ is associated with its fundamental line $(n=1)$, and considering a gravitational redshift of $z=0.3$ at the surface of a typical neutron star having a mass of $1.4 M_{\odot}$ and a radius of $10 \mathrm{~km}$. From the measurements of the centroid energy at $10.81_{-0.49}^{+0.48} \mathrm{keV}$, for the phase averaged case, we could thus establish the strength of the magnetic field of the pulsar as $\left(1.21_{-0.06}^{+0.05}\right) \times 10^{12} \mathrm{G}$. Considering the variation in energy of first cyclotron line with pulse phase we can get a range of (9.7-14.4) keV after including the error bars. This would translate to a variation in the magnetic field strength of $(1.1-1.6) \times 10^{12} \mathrm{G}$ over the pulse phase of the pulsar. The strength of the magnetic field derived for GRO J2058+42 from its spectrum is comparable to the magnitude of those measured for other X-ray pulsars, such as KS 1947+300 (12.2 keV) (Furst et al. 2014), Swift J1626.3-5156 (10 $\mathrm{keV}$ ) (DeCesar et al. 2013) and 4U 0115+63 (12 keV) (Jun et al. 2012; Ferrigno et al. 2009) where respective energy values of their fundamental cyclotron absorption features are shown within parenthesis.

\section{CONCLUSION}

AstroSat observed the Be-binary pulsar GRO J2058+42 on April 10, 2019 during its latest long outburst between March-May 2019, using both LAXPC and SXT instruments for a total exposure of $57 \mathrm{ks}$. The source intensity during this observation had declined to $170 \mathrm{mCrab}$, about $66 \%$ of its peak intensity of $256 \mathrm{mCrab}$. AstroSat observation showed a clear detection of strong pulsation from the source. The spin-period of the pulsar was determined as $194.2201 \pm 0.0016$ $\mathrm{s}$ and its average spin-up rate was $\dot{\nu}=(1.65 \pm 0.06) \times 10^{-11} \mathrm{~Hz} \mathrm{~s}^{-1}$ corresponding to MJD 58583.10868148. Pulse-profiles derived at different energy bands covering 3-80 keV, showed pronounced and multiple pulse structure, which showed 
a variation in shape and pulse-fraction with energy. The RMS pulse fraction varied from about $20 \%$ to $30 \%$ between 3-20 keV, beyond which it was approximately constant. Pulse-profiles derived from recent AstroSat observations were found to be similar in shape to those reported from RXTE observations, at a relatively lower source intensity by an order of magnitude. Thus, the source did not show drastic change in its pulse shape and pulse-fraction within the observed variation of source luminosity.

A broad QPO feature corresponding to a frequency of $0.090 \mathrm{~Hz}$ was detected for the first time from AstroSat observations. This provided an evidence for the formation of a transient accretion disk around the neutron star. The QPO, therefore, offered us a tool to probe inner region of the accretion disk and to quantify the transfer of angular momentum through such accretion disk. This enabled us to estimate the torque applied to the neutron star by mass transfer from a transient accretion disk. This resulted in the prediction and determination of observed change in spinperiod of the pulsar by 2.3 seconds during the recent 46 days outburst. Similar change in spin-period was observed by BATSE during the earlier outburst in 1995 and also by the Fermi-GBM during this latest outburst. This therefore, favors a scenario where pulsar could be effectively spun-up by such a magnitude, in a short span of 46 days.

The centroid energy of cyclotron line and its harmonics were found to be consistent within errors for the two models used for spectral fits. The line energies are found to vary within the range of (9.7-14.4) keV, (19.3-23.8) keV and (37.843.1) keV respectively for the observed 3 absorption features. The detection of cyclotron line lead to determination of strength of its strong magnetic field of the neutron star. Therefore, using AstroSat observation, we could establish the strength of magnetic field of the pulsar as $(1.1-1.6) \times 10^{12}$ G. Further observations are required to study spectral variation with source luminosity, variation of magnetic field strength during its long outburst duration and with time, to probe the nature of its accretion column and geometry where cyclotron lines are produced.

\section{ACKNOWLEDGMENTS}

We gratefully acknowledge all the support received from the Indian Space Research Organization (ISRO) for successful realization of AstroSat mission from the initial phase of instrument building, tests and qualifications to software developments and mission operations. We also acknowledge the support received from the LAXPC Payload Operation Center (POC), TIFR, Mumbai for the release of verified data, calibration data products and pipeline processing tools. This work has also utilized the data from the Soft X-ray Telescope (SXT) and hence thankfully acknowledge SXT POC at TIFR for releasing the data through the ISSDC data archive center and providing the necessary software tools. We acknowledge software engineers Sanket Kotak, Ashutosh Bajpai and Harshal Pawar, for their vital services in software development activities and timely completion of the full SXT pipeline processing chain along with relevant documentation. We also acknowledge the support of the Department of Atomic Energy, Government of India, under project no. 12-R\&D-TFR-5.02-0200. This research has also made use of data obtained from Swift-BAT and RXTE through the High Energy Astrophysics Science Archive Research Center On-line Services, provided by the NASA/Goddard Space Flight Center, we acknowledge their vital support. We also thank Fermi-GBM team of NASA/MSFC for sharing monitoring data of the pulsar period measurements of this source during its outburst. We also acknowledge generous support of NASA's HEASARC for offering all the useful software and tools for analysis of Astronomical data. We thank an anonymous Referee for critical comments, which have improved the manuscript significantly.

Facility: Astrosat

\section{REFERENCES}

Alpar, M. A. \& Shaham, J. 1985, Nature, 316, 239

Angelini L., Stella L. \& Parmar A. N., 1989, ApJ, 346, 906

Antia, H. M. et al. 2017 ApJS, 231, 10

Barthelmy, S. D., et al. 2019, GCN-23985, March 22, 2019

Barret, D. \& Vaughan, S. 2012, ApJ, 746, 131B.

Bellm, E.C. et al. 2014, ApJ, 792, 108.

Bhalerao, V. et al. 2015, MNRAS, 447, 2274-2281

Bildsten, L. et al. 1997, ApJS, 113, 367.
Bodaghee, A. et al. 2016, ApJ, 823, 146.

Brejnholt, N. F. et al. 2012, SPIE, vol 8843.

Coburn, W. 2001, PhD. Thesis.

Coburn, W. et al. 2002, ApJ, 580, 394.

Burderi, L. et al. 2000, ApJ, 530, 429.

DeCesar, M. E., et al. 2013 APJ, 762, 61

Devasia, J., et al. 2011 MNRAS, 414, 1023

Dugair, M. R., et al. 2013 MNRAS, 434, 2458 
Enoto T. et al. 2008, PASJ, 60, S57

Ferinelli R. et al. 2008, ApJ, 680, 602

Ferrigno C. et al. 2009, A\&A, 498, 852

Finger, M. H., Wilson, R. B., \& Harmon, B. A. 1996, ApJ, 459, 288

Finger, M. H., 1998, Adv. Space Res. vol 22, No.7, 1007

Frank, J., King, A., \& Raine, D. J. 2002 Accretion Power in Astrophysics, pp. 398. ISBN 0521620538. Cambridge, UK: Cambridge University Press, February 2002

Furst F. et al. 2014, ApJ, 784, L40.

Ghosh, P. 1996, ApJ, 459, p 244-248.

Ghosh, P. 1998, Adv. Space Research, vol 22, No 7, p 1017-1024.

Harison, F. A. et al. 2013, ApJ, 770, 103.

Heindl, W. A. et al. 2004, in AIP Conf. Proc.714, X-RAY TIMING 2003: Rossie and Beyond, ed. P. Kaaret, F. K. Lamb, \& J. H. Swank (Melville, NY: AIP), 323

Heindl, W. A. et al. 1999, ApJ, 521, L49-53.

In't Zand, J. J. M., Baykal, A., \& Strohmayer, T. E. 1998, ApJ, 496, 386.

Jaiswal, G. K. et al. 2015, MNRAS, 453, L21-25.

Jun, L. et al. 2012, MNRAS, 423, 2854

Kennea, J. A. et al. 2019, GCN-24021, March 27, 2019

Klochkov, D. et al., 2008, A\&A 482,907

Kreykenbohm, I. et al., 2002, A\&A 395, 129

Kreykenbohm, I. et al., 2004, A\&A 427, 975

Kreykenbohm, I. et al., 2005, A\&A 433, L45

Krimm, H. A. et al., 2013, ApJSS 209, 14

La Barbera, A., et al. 2001 ApJ, 553, 375

La Barbera, A., et al. 2003 A\&A, 400, 993

Maitra, C. 2016, J Ap. Astr., 123, 23

Malacaria, C., Jenke, P., Wilson-Hodge, C. A., \& Roberts, O. J. 2019, ATel 12614, March 28, 2019

McBride V. A. et al. 2007, A\&A, 470, 1065.

Meszaros, P. 1992, High-Energy Radiation from Magnetized Neutron Stars (Chicago, IL: Univ. Chicago Press)

Molkov, S., Lutovinov, A., Tsygankov, S., Mereminskiy, I., \& Mushtukov, A. 2019, ApJ, 883, L11

Mukerjee, K., Agrawal, P. C. et al. 2000, A\&A, 353, 239

Mukerjee, K., Agrawal, P. C. et al. 2001, ApJ, 548, 368

Mushtukov, A. A. 2018, MNRAS, 474, 5425

Nakajima, M. 2010, ApJ, 710, 1755

Nespoli, E. \& Reig, P. 2011, A\&A, 526, A7

Nishimura, O. 2003, PASJ, 55,849

Nishimura, O. 2005, PASJ, 57, 769

Nishimura, O. 2013, PASJ, 65,84

Nishimura, O. 2015, ApJ, 807, 164

Nishimura, O. 2019, PASJ, 71(2), 42(1-9)
Okazaki A. T. and Negueruela I. 2001, A\&A, 377, 161

Okazaki A. T., Bate M. R., Ogilvie G. I. and Pringle J. E. 2002, MNRAS, 337, 967

Okazaki A. T. 2016, APS Conference series, Vol 506, 1

Orlandini, M. et al. 2012, ApJ, 748, 86

Parmar, A., et al. 1989, ApJ, 338, 373

Paul, B., \& Rao, A. R. 1998, A\&A, 337, 815

Paul, B., et al. 2001, A\&A 370, 529

Protassov, R., et al. 2002, ApJ 571, 545

Pottschmidt, K., et al. 2005, ApJ 634, L97

Poutanen, J., et al. 2013, ApJ 777, 115

Qu L. J.. et al. 2005, ApJ, 629, L33-L36

Reig, P., 2011 Ap \& SS 332, issue 1, p 1-29

Reig, P., Negueruela I., Papamastorakis, G., Manousakis, A.\& Kougentakis, T., 2005 A\&A 440, 637-646

Schwarm, F.-W. et al. 2017, A\&A 601, A99

Singh, K. P., et al. 2016, Proceedings of the SPIE, Volume 9905, id. 99051E 10 pp.

Soong, Y., \& Swank, J. H. 1989, Proc. 23rd ESLAB Symp. on Two Topics in X-ray Astronomy, eds. J. Hunt, \& B. Battrick, ESASP 296..617S

Soong, Y., et al. 1990, ApJ, 348, 641

Staubert, R., Trumper, J., Kendziorra, E., et al. 2019, A\&A, 622, A61

Suchy, S., et al. 2008, ApJ, 675, 1487

Suchy, S., et al. 2012, ApJ, 745, 124

Takeshima, T., Dotani, T., Mitsuda, K. \& Nagase, F. 1994, ApJ, 436, 871-874

Takeshima, T. 1998, Proc. IAU Symposium 188, Ed. K. Koyama, S. Kitamoto, M. Itoh, Kluwer Academic, Dordrecht, p. 368

Tanaka, Y. 1986, in IAU Colloq. 89: Radiation Hydrodynamics in Stars and Compact Objects, eds. D. Mihalas \& K.-H. A. Winkler, 198

Titarchuk, L. 1994, ApJ 434, 570

Truemper, L. et al. 1978, ApJ 219, L105-L110

Tsygankov, S. S. 2010, MNRAS, 401, 1628

van der Klis, M., Stella, L., White, N., Jansen, F., \&

Parmar, A. N. 1987, ApJ, 316, 411

van der Klis, M. 2000, Annu. Rev. Astron. Astrophys. 38:717-760

Varun et al. 2019, ApJ, 880, 61

Wang, D. H., Chen, L., Zhang, C. M., Lei, Y. J., and Qu, J.

L., 2014, Astronomische Nachrichten 335, 168-177

Voges, W. et al. 1982, ApJ, 263,803

Vybornov, V. et al. 2017, A\&A 601, A126

Wilson, C. A., Finger, M. H. Harmon, B. A., Chakrabarty, D., \& Strohmayer, T. 1998, ApJ 499, 820

Wilson, C. A. et al. 2005, ApJ 622, 1024 